\title{
Epithelial splicing regulatory protein 2-mediated alternative splicing reprograms hepatocytes in severe alcoholic hepatitis
}

\author{
Jeongeun Hyun, ${ }^{1,2,3}$ Zhaoli Sun, ${ }^{4}$ Ali Reza Ahmadi, ${ }^{4}$ Sushant Bangru, $, 5,6$ Ullas V. Chembazhi, ${ }^{5}$ Kuo Du, ${ }^{1}$ Tianyi Chen, ${ }^{7}$ \\ Hidekazu Tsukamoto, ${ }^{8,9}$ Ivan Rusyn, ${ }^{10}$ Auinash Kalsotra, ${ }^{5,6,11}$ and Anna Mae Diehl ${ }^{1}$
}

'Department of Medicine, Duke University Health System, Durham, North Carolina, USA. ²Regeneration Next, Duke University School of Medicine, Durham, North Carolina, USA. ${ }^{3}$ Institute of Tissue Regeneration Engineering (ITREN) and College of Science and Technology, Dankook University, Cheonan, South Korea. ${ }^{4}$ Department of Surgery, Johns Hopkins University School of Medicine, Baltimore, Maryland, USA. ${ }^{5}$ Department of Biochemistry, School of Molecular and Cellular Biology, and ${ }^{6}$ Cancer Center at Illinois, Beckman Institute for Advanced Science and Technology, University of Illinois at Urbana-Champaign, Urbana, Illinois, USA. 'Department of Molecular Cenetics and Microbiology, Duke University, Durham, North Carolina, USA. ${ }^{8}$ Southern California Research Center for ALPD and Cirrhosis and Department of Pathology, Keck School of Medicine, University of Southern California, Los Angeles, USA. 'Veterans Affairs Greater Los Angeles Healthcare System, Los Angeles, California, USA. ${ }^{10}$ Department of Veterinary Integrative Biosciences, Texas A\&M University, College Station, Texas, USA. "'Carl R. Woese Institute for Cenomic Biology, University of Illinois at Urbana-Champaign, Urbana, Illinois, USA.

\begin{abstract}
Severe alcoholic hepatitis (SAH) is a deadly liver disease without an effective medical therapy. Although SAH mortality is known to correlate with hepatic accumulation of immature liver cells, why this occurs and how it causes death are unclear. Here, we demonstrate that expression of epithelial splicing regulatory protein 2 (ESRP2), an RNA-splicing factor that maintains the nonproliferative, mature phenotype of adult hepatocytes, was suppressed in both human SAH and various mouse models of SAH in parallel with the severity of alcohol consumption and liver damage. Inflammatory cytokines released by excessive alcohol ingestion reprogrammed adult hepatocytes into proliferative, fetal-like cells by suppressing ESRP2. Sustained loss of ESRP2 permitted reemergence of a fetal RNA-splicing program that attenuates the Hippo signaling pathway and thus allows fetal transcriptional regulators to accumulate in adult liver. We further showed that depleting ESRP2 in mice exacerbated alcohol-induced steatohepatitis, enabling surviving hepatocytes to shed adult hepatocyte functions and become more regenerative, but threatening overall survival by populating the liver with functionally immature hepatocytes. Our findings revealed a mechanism that explains why liver failure develops in patients with the clinical syndrome of SAH, suggesting that recovery from SAH might be improved by limiting adult-to-fetal reprogramming in hepatocytes.
\end{abstract}

\section{Introduction}

Severe alcoholic hepatitis (SAH) is an alcohol-related liver disease with a short-term mortality rate approaching $50 \%$ and a propensity for rapid progression to cirrhosis in survivors (1-3). The major causes of early death in SAH are hepatic failure and infection (2-4). Previous studies have shown that short-term mortality in SAH correlates with the accumulation of proliferative liver cells that express stem/ progenitor cell markers via a process called ductular reaction (5-7). Hence, fatal liver failure in SAH may be caused by excessive repopulation of the liver by fetal-like proliferative liver cells that lack mature hepatocyte functions; thus, inhibiting their accumulation would be therapeutic. However, this is not yet feasible because what causes fetal-like cells to accumulate in the livers of SAH patients is unknown.

Single-cell gene expression analysis recently demonstrated that feeding alcohol to adult rats increased the proportion of hepatocytes that are either becoming proliferative or proliferat-

Conflict of interest: The authors have declared that no conflict of interest exists. Copyright: ( 2020, American Society for Clinical Investigation.

Submitted: August 14, 2019; Accepted: January 14, 2020; Published: March 16, 2020.

Reference information: J Clin Invest. 2020;130(4):2129-2145.

https://doi.org/10.1172/JCl132691. ing (8), suggesting that excessive alcohol drinking might promote some degree of hepatocyte dedifferentiation. Maintaining hepatocytes in a differentiated state is a critical function of the Hippo/ YAP signaling pathway (9). Hippo signaling promotes phosphorylation and degradation of YAP and TAZ, transcriptional regulators that drive organ growth during fetal development and promote cell proliferation and epithelial-mesenchymal transitions (EMT) essential for adult tissue regeneration (10). In healthy adult livers, Hippo signaling activity is high, keeping YAP/TAZ inactive and permitting mature hepatocyte transcription factors, such as $\mathrm{C} /$ $\mathrm{EBP} \alpha$ (11), to control gene expression. In contrast, Hippo signaling activity is low in fetal livers, and this enables YAP/TAZ activity. Experiments in mice have shown that deleting $N f 2$, a component of the Hippo signaling pathway, or directly activating YAP in adult hepatocytes causes them to dedifferentiate into proliferative ductal-like cells (9). Reintroducing Hippo signaling reverses this process and permits the reprogrammed hepatocytes to differentiate back to their basal nonreplicative phenotype (9).

It was shown that the activity of 2 key components of the Hippo signaling pathway, $N f 2$ and Csnk1d, is controlled by an adult RNA-splicing factor, epithelial splicing regulatory protein 2 (ESRP2) (12). By retaining exons to generate longer adult mRNAs, 
A
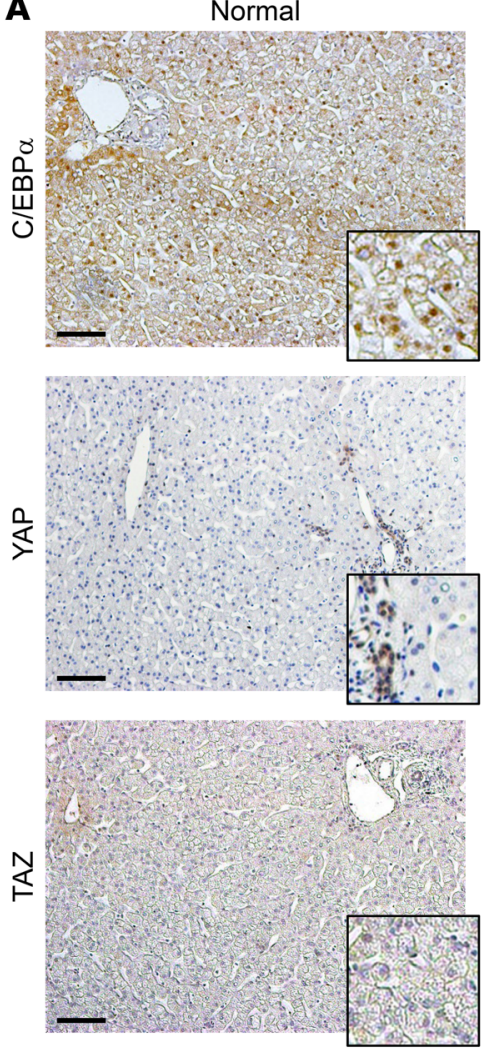

B

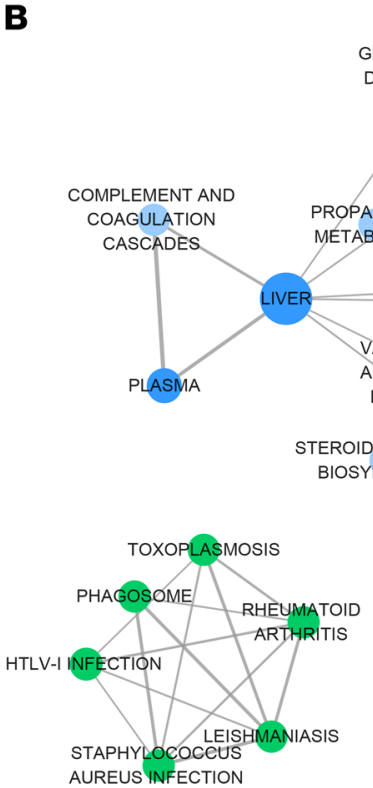

ESRP2 converts the fetal splicing variants that encode low kinase activity NF2 and CSNK1D proteins into adult splicing variants that encode kinases with higher activity to suppress YAP/TAZ (12). ESRP2 is absent in fetal livers, but begins to be expressed in hepatocytes shortly after birth (13). It is critically important for switching on the adult splicing program in approximately $20 \%$ of hepatocyte mRNAs, generating splicing variants that encode functional differences in multiple proteins (13). Importantly, ESRP2 targets critically control hepatocyte proliferative activity because adult ESRP2-KO mice demonstrate significant-
Figure 1. Hepatocytes are fetal like in patients with SAH. (A) IHC for C/EBP $\alpha, Y A P$, and TAZ in explanted liver tissues of patients with $\mathrm{SAH}$ and healthy human liver. Representative images are shown. Scale bars: $100 \mu \mathrm{m}$. Original magnification, $\times 100 ; \times 400$ (higher magnification images). (B) An enrichment map consisted of KEGG pathways and annotations of tissue expression that are involved by significantly upregulated (green circles) or downregulated (blue circles) genes in liver tissues of SAH patients compared with healthy controls ( $n=5$ each) by RNA-Seq analysis. The node size is proportionate to the number of genes for each category, and thickness of edges is proportionate to the number of shared genes between categories. ly increased rates of hepatocyte proliferation (13). ESRP2 is also known to be downregulated during $\operatorname{EMT}(14,15)$, permitting fetal splicing variants of EMT-related genes to accumulate.

The potential role of ESRP2 as a key regulator of hepatocyte maturation is supported by the finding that expression of ESRP2 is suppressed, as nonreplicating mature hepatocytes transition to become proliferative after $70 \%$ partial hepatectomy $(\mathrm{PH})$, but promptly recover back to baseline as regeneration is completed (16). Indeed, hepatocytes that are becoming proliferative accumulate excessively in livers of alcohol-fed rats after $\mathrm{PH}$, and alcohol 
A

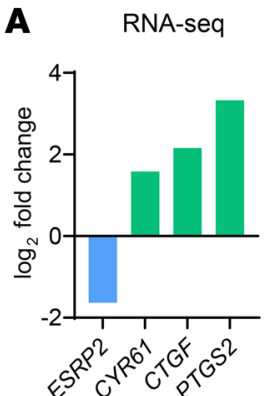

B

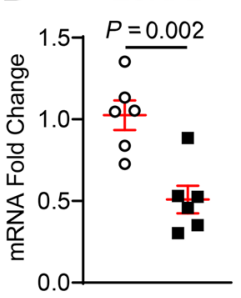

AREG

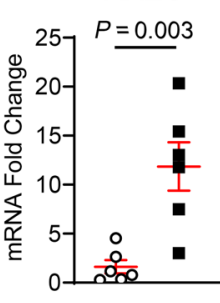

CYR61

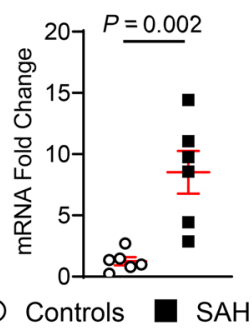

CTGF

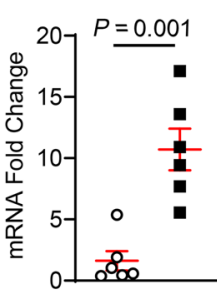

PTGS2

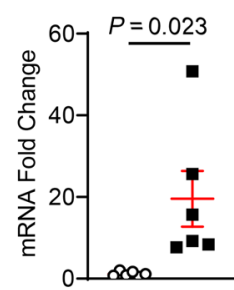

C

Controls

ESRP2

GAPDH

E

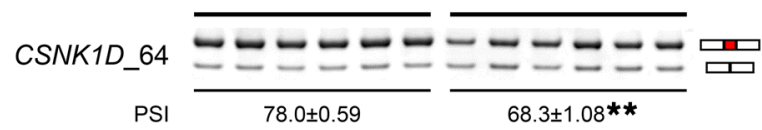

NF2_45 ヒニニニニニニニニニニニ

PSI $67.3 \pm 1.67 \quad \frac{50.3 \pm 1.78 * *}{2}$

SLK 93 レーーーーー-

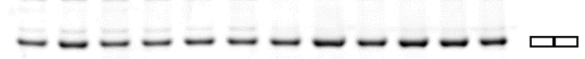

PSI

$60.4 \pm 1.63$

$34.9 \pm 3.17 * \star$

FLNB_72 39

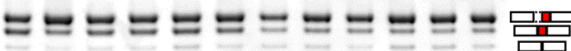

$32.6 \pm 1.87$

$22.4 \pm 1.12$ *

H

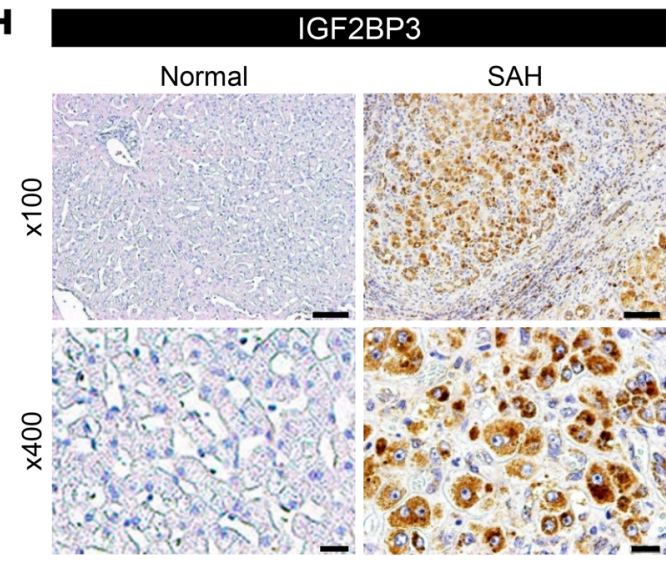

Figure 2. ESRP2 expression and ESRP2-mediated adult RNA-splicing program are downregulated in livers of patients with SAH. (A) The log ${ }_{2}$ fold changes of ESRP2 and direct YAP/TAZ target genes (CYR61, CTCF, PTCS2) assessed by RNA-Seq in liver explants from SAH patients relative to healthy controls ( $n=5$ each). (B) Quantitative reverse transcription PCR (qRT-PCR) analysis for ESRP2 and YAP/TAZ target genes (AREG, CYR61, CTCF, PTCS2) in human SAH livers and healthy controls. (C) Immunoblots for ESRP2 normalized to GAPDH as a loading control in lysates of liver tissues from SAH patients and healthy controls. (D) IHC for ESRP2 in explanted liver tissues of SAH patients and healthy human liver. (E) Alternative splicing of ESRP2 target mRNAs including CSNK1D, NF2, SLK, and FLNB between liver tissues of SAH patients and healthy controls. Upper bands include the exon (red part), while the lower bands skip the exon. Exon lengths are shown beside the gene name, and the differences in PSI values are shown as mean \pm SEM below each image. $(\mathbf{F}-\mathbf{H})$ qRT-PCR analysis $(\mathbf{F})$, immunoblot $(\mathbf{G})$, and IHC $(\mathbf{H})$ for IGF2BP3 in the livers of SAH patients and healthy controls. qRT-PCR results are graphed as dot plots (SAH, black squares; healthy controls, white circles) showing mean \pm SEM (red bars) ( $n=5$ individuals/group). See full, unedited blots for $\mathbf{C}$ and $\mathbf{G}$ and full, unedited gels for $\mathbf{E}$ in the supplemental material. Statistical analysis was performed by using 2-tailed Student's $t$ test between 2 groups ( $n=$ 5 individuals/group, $P$ values are specified otherwise, ${ }^{* *} P<0.001$ ). Representative images are shown for IHC. Scale bars: $100 \mu \mathrm{m}$ (original magnification, $\times 100$ ), $20 \mu \mathrm{m}$ (original magnification, $\times 400$ ).

prevented maturation of hepatocytes (8), which explains why liver regeneration is inhibited by alcohol $(17,18)$. However, the mechanisms involved were not defined. The present study evaluates the hypothesis that the ESRP2-mediated adult splicing program is inactivated in some individuals with alcohol-induced liver injury, causing extensive repopulation of SAH livers with immature, fetal-like cells. The results identify the mechanism for liver failure in SAH by revealing that TNF- $\alpha$ and IL-1 $\beta$ (19), proinflamma- 
tory cytokines that accumulate in alcohol-injured livers, suppress ESRP2 in adult hepatocytes. Loss of ESRP2 permits relatively inactive fetal splicing variants of Hippo kinases (HKs) to accumulate and enables the reactivation of YAP/TAZ, which then reprogram hepatocytes to become more proliferative but less capable of performing vital, mature hepatocyte functions.

\section{Results}

Adult-to-fetal reprogramming occurs in livers of patients with SAH. To determine whether adult-to-fetal reprogramming occurs in human livers during the pathogenesis of $\mathrm{SAH}$, we leveraged knowledge about transcription factor switching during normal development. As the liver matures, progressive activation of adult hepatocyte transcription factors, such as $\mathrm{C} / \mathrm{EBP} \alpha$, suppresses the expression of genes that promote hepatocyte proliferation, but induces the expression of genes that accomplish mature liver functions (11). Conversely, YAP and TAZ, transcriptional regulators that are active in fetal livers, become silenced by progressive increases in Hippo signaling activity as the liver matures. Thus, C/EBP $\alpha$ localizes within nuclei of healthy adult hepatocytes, but YAP and TAZ are barely detectable (Figure 1A). In contrast, almost none of the hepatocyte nuclei expressed $\mathrm{C} / \mathrm{EBP} \alpha$, but both YAP and TAZ were abundant in explanted livers of adult patients who underwent liver transplantation for SAH (Figure 1A), suggesting that hepatocytes dedifferentiate during SAH. Since YAP and TAZ have been shown to induce stem cell characteristics and EMT (10), we also examined the expression of 2 liver progenitor markers, TNFRSF12A (also known as FN14) (20) and SOX9 (21), and 2 important EMT-related factors, SMAD3 and CDH1 (also known as E-cadherin) (15). Although TNFRSF12A, SOX9, and the EMT inducer SMAD3 are rarely detected in healthy liver, livers of SAH patients are enriched with cells expressing these progenitor and mesenchymal markers. Conversely, CDH1, an adherens junction protein that maintains epithelial integrity in healthy liver (22), is lost from hepatocytes of SAH patients (Supplemental Figure 1; supplemental material available online with this article; https://doi.org/10.1172/JCI132691DS1).

To more globally assess SAH-associated changes in liver cell phenotypes, we performed Kyoto Encyclopedia of Genes and Genomes (KEGG) pathway analysis using transcriptome profiles obtained by RNA sequencing (RNA-Seq) of explanted livers of 5 SAH patients and 5 controls without known liver disease. Genes responsible for mature hepatocyte functions, including synthesis of clotting factors and multiple metabolic pathways, were suppressed in SAH livers (Figure 1B and Supplemental Figure 2). In contrast, the Notch pathway, which drives cells in developing livers to become ductal like (23), is upregulated, as are genes involved in cancer and chemokine signaling pathways (Figure 1B and Supplemental Figure 2). These findings support the concept that adult-to-fetal reprogramming occurs in human SAH livers.

ESRP2-controlled adult RNA-splicing program is suppressed in human SAH. Recently, we discovered that ESRP2 controls the adult splicing program of mRNAs that encode 2 key components of the HK cascade, CSNK1D and NF2, preserving their activities to phosphorylate and degrade YAP and TAZ in mature hepatocytes (12). Therefore, we examined these ESRP2-mediated splicing events to address how YAP and TAZ are reactivated in liver tissues of adult SAH patients. We found that expression of ESRP2 was sig- nificantly downregulated at both the levels of mRNA and protein in livers of SAH patients compared with healthy livers (Figure 2, A-D). In addition, ESRP2 was mislocalized, being restricted to cell cytosol in SAH livers while localizing in hepatocyte nuclei of the healthy liver (Figure 2D). The loss of ESRP2 in SAH livers was associated with accumulation of smaller (fetal) splicing variants of the HK mRNAs, CSNK1D and NF2, at the expense of longer (adult) splicing variants of these transcripts (Figure $2 \mathrm{E}$ ), as evidenced by a fall in the percentages of total transcripts with exons spliced in (percentage spliced in [PSI]) (Figure 2E). Consistent with reduced kinase activity of HKs encoded by fetal CSNK1D and NF2, YAP and TAZ activity is increased in SAH livers, as demonstrated by upregulation of YAP/TAZ direct target genes (AREG [ref. 24], CYR61 [ref. 25], CTGF [ref. 26, 27], PTGS2 [ref. 28]) (Figure 2, A and B). Fetal splicing variants of SLK and FLNB, 2 additional ESRP2 targets that encode proteins involved in EMT (29-31), also accumulated in SAH livers (Figure 2E). These results demonstrate that ESRP2 simultaneously regulates multiple targets to enrich adult hepatocytes with proteins that they require to accomplish vital liver-specific functions while limiting the production of proteins involved in proliferation, migration, and morphogenesis, functions that are unnecessary in healthy adult livers.

Interestingly, in human SAH, downregulation of ESRP2 (an adult RNA-binding protein [RBP]) was associated with upregulation of a YAP-dependent fetal RBP that promotes stem/progenitor-like traits in liver cells, insulin-like growth factor-2 mRNA-binding protein 3 (IGF2BP3) (ref. 16 and Figure 2, F-H). Whether cells exhibit fetal or adult phenotypes is controlled by families of coordinately regulated RBPs that regulate the stability, translation, and splicing of mRNAs. Together, these data indicate that adult hepatocytes are reprogrammed to become more fetal like during SAH and suggest that this dedifferentiation process is mediated, at least in part, by reactivation of a fetal RNA-splicing program that emerges when the adult splicing factor, ESRP2, is suppressed.

Adult-to-fetal reprogramming responses correlate with the severity of inflammatory liver damage in mouse models of alcoholic liver disease. Since preclinical models are necessary to determine whether and how misregulated hepatocyte reprogramming causes liver failure, we screened liver samples from different mouse models of alcoholic hepatitis (AH) to assess whether these models recapitulate the adult-to-fetal reprogramming that occurs in human SAH livers. We demonstrated fetal reprogramming responses reminiscent of those observed in human SAH in the Tsukamoto-French mouse model, which uses a protracted highfat $(\mathrm{HF})$ diet/intragastric ethanol infusion protocol to generate severe inflammatory liver injury and moderate liver fibrosis (ref. 32 and Supplemental Figure 3). This AH model suppresses Esrp2 expression, promotes relative accumulation of fetal-splicing (versus adult splicing) variants of mRNAs encoding both HKs and genes controlling cell proliferation and differentiation (Slk and Kras; ref. 13), and increases YAP/TAZ activity, as evidenced by induction of their target genes, including Areg, Birc5 (33), and Itgb2 (ref. 26 and Supplemental Figure 3).

We found that some of the human SAH-like reprogramming responses also occur in 3 other models of alcohol-induced liver injury: (a) the Gao binge model of chronic (10 days) plus acute alcohol binging (also known as the National Institute on Alcohol Abuse 

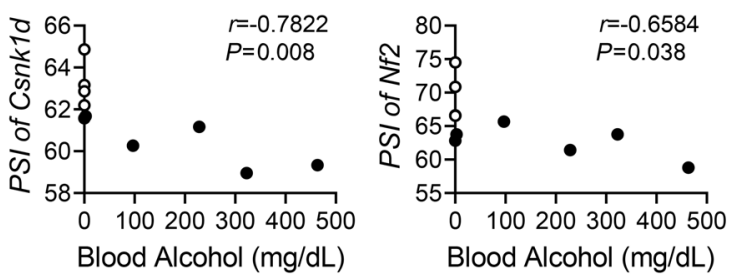

Ctrl
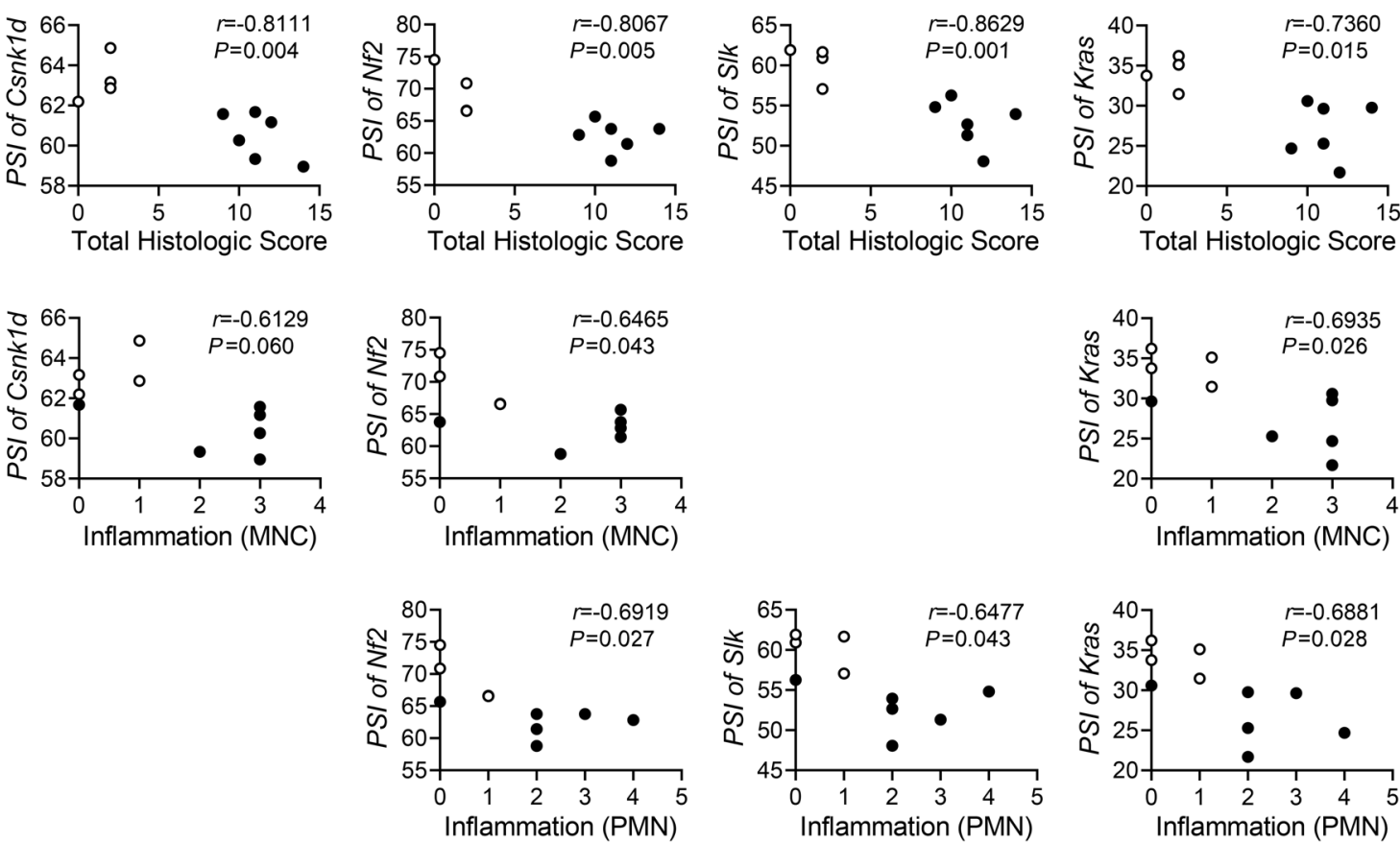

Figure 3. Fetal RNA splicing occurs as increase of alcohol exposure and liver damage in mouse model of AH. The Pearson's $r$ correlation analysis between the PSI of Csnk1d, Nf2, Slk, or Kras in liver tissues and the levels of blood alcohol, total histologic score, or inflammation of control (Ctrl) or AH mice of the Tsukamoto-French model ( $r$, correlation coefficient). Results are graphed as dot plots (AH, black squares; controls, white circles). MNC, mononuclear cell; PMN, polymorphonuclear neutrophil.

and Alcoholism [NIAAA] model, refs. 34, 35); (b) the Lieber DeCarli HF diet model of voluntary chronic alcohol diet consumption followed by binging (5); and (c) the long-term HF/3,5-diethoxycarbonyl-1,4-dihydrocollidine (HF/DDC) diet plus alcohol feeding model (36). In these models, alcohol feeding promotes suppression of Esrp2 and adult splicing variants relative to pair-fed or HF/ DDC diet-fed control mice (Supplemental Figure 4). However, liver injury is much milder in these models than in the Tsukamoto-French SAH model or human SAH. Effects on Esrp2, splicing variants (Supplemental Figure 4), and YAP/TAZ target genes are also less profound (data not shown). Therefore, the aggregate data suggest that the levels of ESRP2 suppression and adult-to-fetal reprogramming parallel the severity of liver injury (and thus the level of regenerative challenge). Further, the PSI indexes inversely correlate with the levels of blood alcohol and the histologic severity of liver inflammation in the Tsukamoto-French AH model (Figure 3), suggesting that accumulation of fetal splicing variants might be influenced by tractable factors that drive SAH pathogenesis.

Proinflammatory cytokines reprogram hepatocytes into fetallike cells by suppressing the ESRP2-mediated adult splicing program. Excessive exposure to macrophage-derived proinflammatory cytokines, such as TNF- $\alpha$ and IL- $1 \beta$, is critically involved in SAH pathogenesis (37), and SAH mortality is known to correlate with accumulation of fetal-like liver cells $(5,6)$. Therefore, we designed experiments to determine whether macrophage-derived factors regulate Esrp2 expression in hepatocytes. We cocultured AML12 adult mouse hepatocytes with RAW264.7 mouse macrophages in Transwells and discovered that this both increases hepatocyte exposure to TNF- $\alpha$ and suppresses hepatocyte expression of Esrp2 (Supplemental Figure 5, A and B). In subsequent studies, we treated AML12 monocultures with TNF- $\alpha$, IL- $1 \beta$, or TNF- $\alpha+$ IL- $1 \beta$ and found that these inflammatory cytokines directly suppress Esrp2 expression (Supplemental Figure 5C). Loss of ESRP2 activity is also evident, since both cytokines stimulate the accumulation of fetal splicing variants of HK mRNA (Csnk1d) or EMT-related genes $(S l k, F \ln b)$ (Supplemental Figure 5D), and promote activation of YAP/TAZ, as evidenced by induction of their target gene (Ptgs2) (Supplemental Figure 5E). Importantly, cytokine-induced reprogramming responses associate with increased hepatocyte growth (Supplemental Figure 5F).

TNF- $\alpha$ was recently reported to induce fetal liver cell traits in primary hepatocytes from healthy adult mice, including the ability to propagate ex vivo indefinitely as spheroids (38). Further, when transplanted into recipient mice with injured livers, these 
A

Esrp2
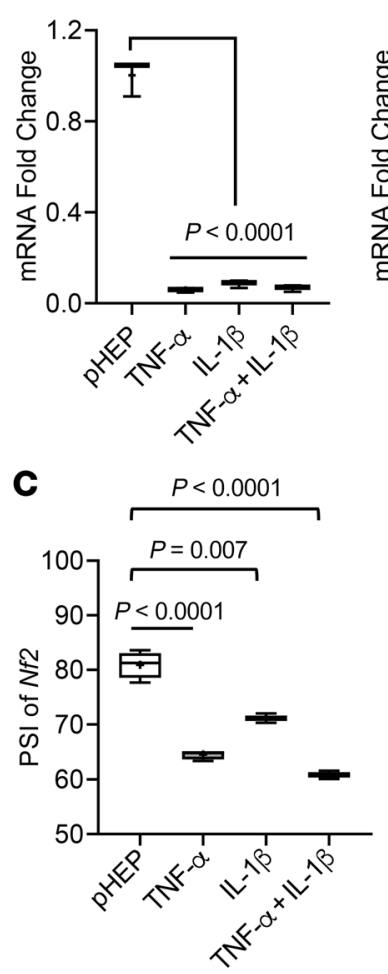

D

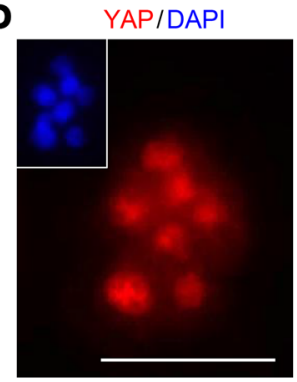

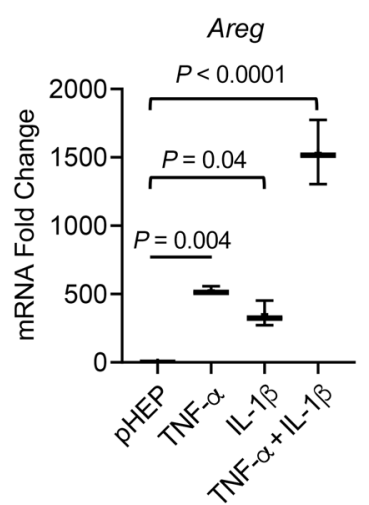

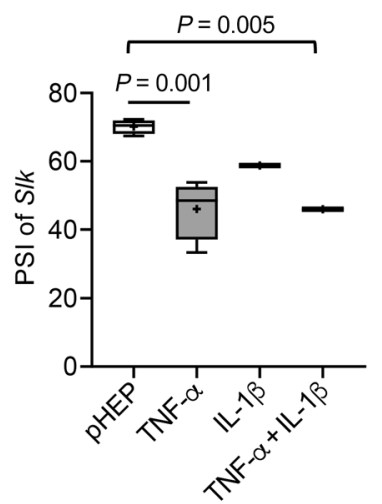

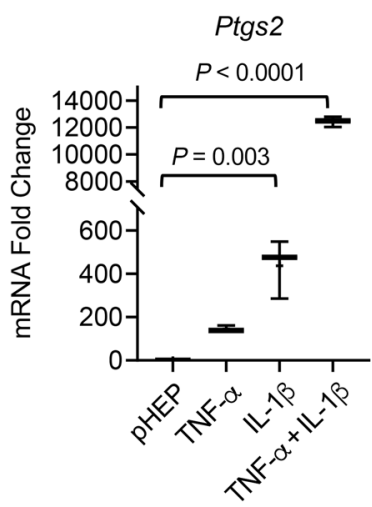

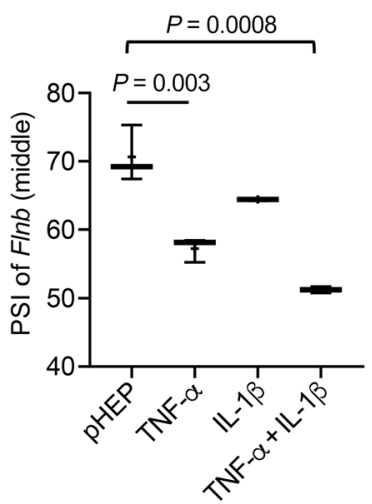

B

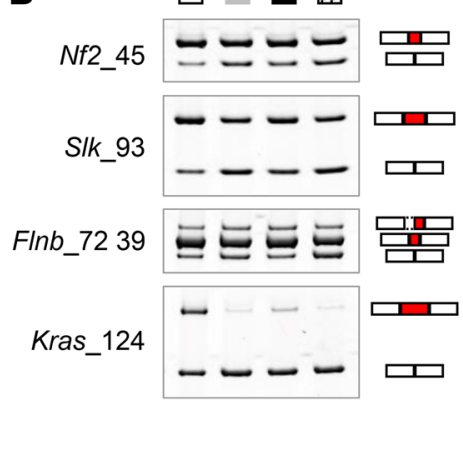

TAZIDAPI

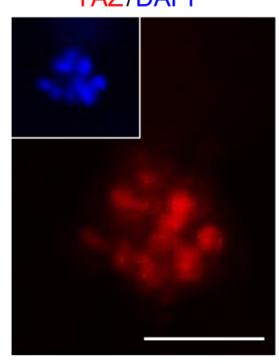

E

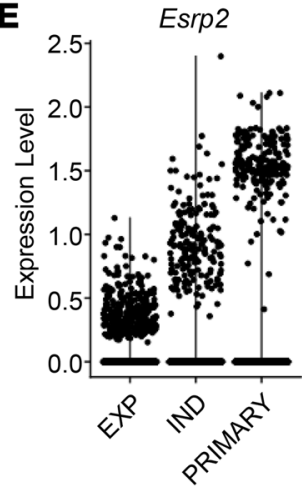

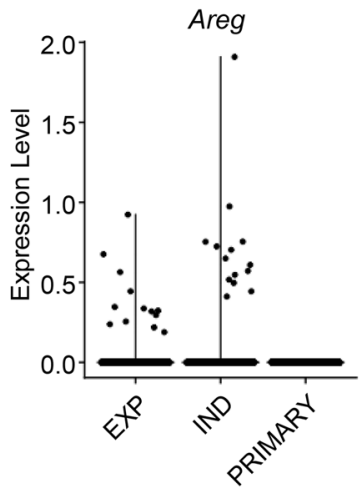

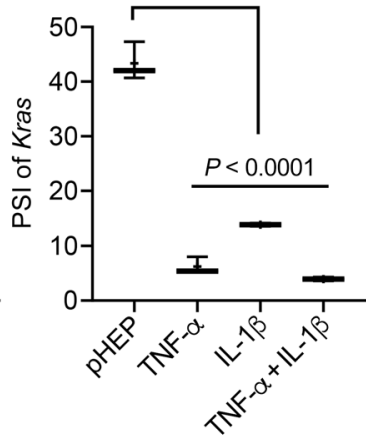

$\square$ pHEP

TNF- $\alpha$

$\mathrm{IL}-1 \beta$

$\square$ TNF- $\alpha+$ IL-1 $\beta$

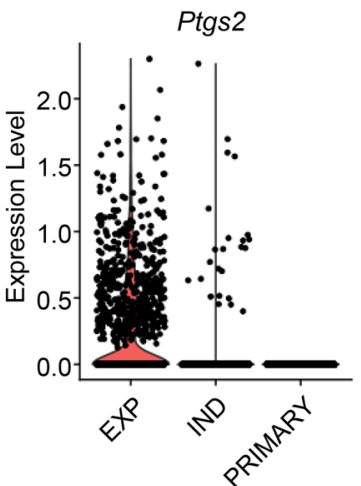

Figure 4. Adult hepatocytes are reprogrammed to fetal-like cells in proinflammatory cytokine-dependent spheroid culture systems. (A) qRT-PCR for Esrp2 and YAP/TAZ target genes (Areg and Ptgs2) in freshly isolated primary mouse hepatocytes (pHEP) and primary hepatocytes cultured under spheroid-forming system for 18 days continuously treated with TNF- $\alpha$, IL-1 $\beta$, or TNF- $\alpha+$ IL-1 1 . Results are graphed as box and whiskers plots (min to max), and means are indicated as plus signs. (B and C) Alternative splicing of Nf2, SIk, Flnb, and Kras in primary hepatocytes as specified. The representative gel images are shown from at least 3 replicates (B). See full, unedited gels in the supplemental material. Results are graphed as box and whiskers plots (min to max), and means are indicated as plus signs (C). (D) Images of hepatocyte spheroids stained for YAP (left, red) and TAZ (right, red) visualized by wholemount immunofluorescence. Nuclear counterstaining was done by DAPI (blue). Scale bars: $50 \mu \mathrm{m}$. (E) scRNA-Seq analysis for Esrp2, Areg, and Ptgs2 in primary hepatocytes (PRIMARY) and hepatocyte spheroids under TNF- $\alpha$-mediated expansion medium (EXP) or TNF- $\alpha$-withdrawn induction medium (IND) as described in a previous publication (38).

fetal-like hepatocytes were shown to repopulate the livers and eventually differentiate into mature, fully functional hepatocytes, proving that "switching" adult-to-fetal/fetal-to-adult programs on and off in hepatocytes is critically important for adult liver regeneration. However, neither the mechanisms driving TNF- $\alpha$-dependent dedifferentiation nor those that permitted hepatocytes to redifferentiate when TNF- $\alpha$ dissipates were described. We used this TNF- $\alpha$-dependent spheroid system to evaluate whether and how growth under these conditions influenced ESRP2-regulated mechanisms controlling adult-to-fetal hepatocyte reprogramming
(Supplemental Figure 6A). Compared with freshly isolated mouse primary hepatocytes, primary adult hepatocytes that were exposed to inflammatory cytokines and grown as spheroids exhibited profound suppression of Esrp2, accumulation of fetal RNA-splicing variants (i.e., decreased PSI), and induction of YAP/TAZ target genes (Figure 4, A-C). Consistent with this finding, immunofluorescent staining showed that both YAP and TAZ translocated into hepatocyte nuclei upon TNF- $\alpha$ and IL-1 $\beta$ stimulation (Figure $4 \mathrm{D}$ and Supplemental Figure 6B). To assess the reproducibility of these results, we analyzed available online single-cell RNA-Seq (scRNA- 
A
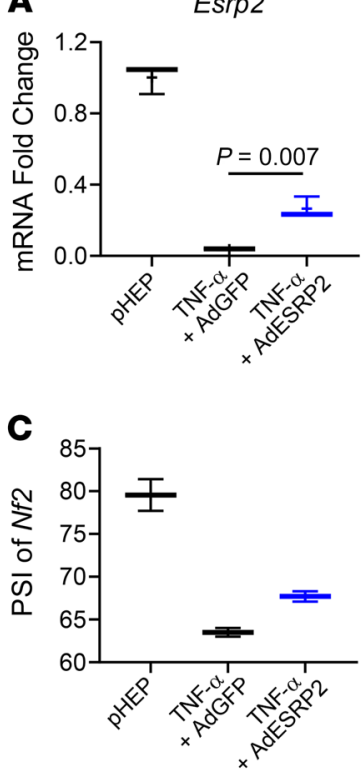
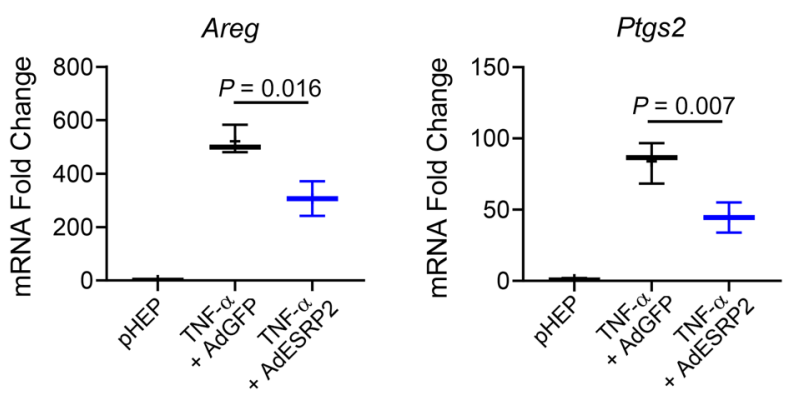

B

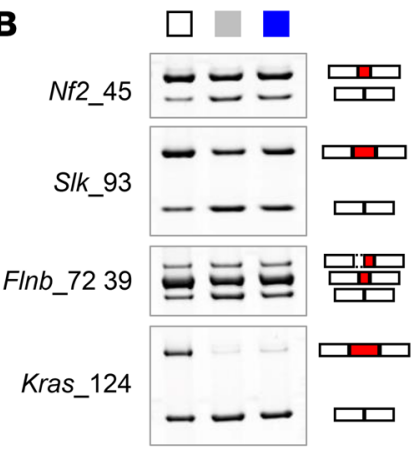

Figure 5. Fetal reprogramming of hepatocytes is mediated by ESRP2-regulated RNA-splicing program. (A) qRT-PCR for Esrp2, Areg, and Ptgs2 in pHEP and TNF- $\alpha$-treated hepatocyte spheroids either transduced with adenoviruses expressing GFP (TNF- $\alpha+$ AdGFP) or mouse ESRP2 (TNF- $\alpha+$ AdESRP2). Results are graphed as box and whiskers plots (min to max), and means are indicated as plus signs. (B and C) Alternative splicing of Nf2, SIk, Flnb, and Kras in primary hepatocytes as specified; pHEP samples and the band images of pHEP are the same as those used for Figure 4B (B). See full, unedited gels in the supplemental material. Results are graphed as box and whiskers plots (min to max), and means are indicated as plus signs (C). All statistical analyses were performed by 1-way ANOVA with Tukey's corrections ( $n=3$ biological replicates/group).

Seq) data that others derived from similar hepatocyte spheroids (38). Freshly isolated primary hepatocytes strongly expressed Esrp2, but transcripts of YAP/TAZ target genes (e.g., Areg or Ptgs2) were not detectable (Figure 4E). In comparison, hepatocytes that were expanding to form spheroids in TNF- $\alpha$-enriched conditions decreased Esrp2 expression but increased the expression of Areg and Ptgs2 (Figure 4E). Remarkably, hepatocyte spheroids recovered levels of Esrp2 expression that approached those of freshly isolated primary hepatocytes after being cultured for 4 days in TNF- $\alpha-$ depleted hepatocyte differentiation-inducing medium (Figure $4 \mathrm{E}$ ). Moreover, none of the hepatocytes that reacquired adult levels of Esrp2 (relative expression level > 1.0) coexpressed YAP/TAZ target genes (e.g., Areg and Ptgs2) (Supplemental Figure 6C).

To investigate whether restoration of ESRP2 in hepatocyte spheroids under TNF- $\alpha$-depleted hepatocyte differentiation-inducing culture conditions suppresses YAP/TAZ activity by retrieving the adult RNA-splicing program, we transduced adenoviruses expressing mouse Esrp2 (AdESRP2) into primary hepatocyte spheroids that we generated using TNF- $\alpha$-dependent conditions. We found that increasing Esrp2 levels promoted accumulation of adult (high PSI) splicing variants of HK mRNAs and EMT-related genes (including Flnb and Kras) (Figure 5, A-C) and decreased YAP/TAZ activity, as evidenced by reduced expression of their target genes (Figure 5A), indicating that TNF- $\alpha$ and IL- $1 \beta$ stimulate hepatocytes to become fetal like by switching off the ESRP2-mediated adult RNA-splicing program.

Excessive accumulation of fetal-like hepatocytes causes liver failure. To assess potential functional consequences of excessive adult-to-fetal reprogramming in injured adult livers, we compared ammonia detoxification in freshly isolated adult hepatocytes and fetal-like hepatocyte spheroids because hyperammonemia is common and predicts acute mortality (39) in humans with SAH and ammonia accumulation promotes hepatic encephalopathy, a defining feature of liver failure in humans $(39,40)$. Healthy adult hepatocytes detoxify ammonia by converting it into either urea (via carbamoyl-phosphate synthase-1 [CPS1]) or glutamine (via glutamate-ammonia ligase [GLUL], also known as glutamine synthetase [GS]) $(41,42)$ in periportal and pericentral areas, respectively (refs. 42, 43, and Figure 6A). The liver-type glutaminase (glutaminase-2 [GLS2]) is activated by ammonia in portal blood and catabolizes glutamine into glutamate, amplifying ammonia concentrations in periportal hepatocytes that then detoxify ammonia through CPS1 and the urea cycle (ref. 43 and Figure 6A). Excess ammonia that is not used for urea synthesis by periportal hepatocytes is taken up by a small population of pericentral hepatocytes (so-called perivenous scavenger cells) that specifically express GS (encoded by the gene GLUL) and convert ammonia into glutamine, thereby preventing ammonia egress into the systemic circulation. Compared with freshly isolated primary hepatocytes, hepatocytes that are reprogrammed to become fetal like and spheroid forming by proinflammatory cytokines (TNF- $\alpha$, IL- $1 \beta$, or TNF- $\alpha+$ IL-1 $\beta$ ) express significantly lower levels of Cps1 and Glul (Figure 6B). Accordingly, conditioned medium from the hepatocyte spheroids contains significantly lower levels of urea and glutamine and considerably higher levels of ammonia than conditioned medium from primary hepatocytes (Figure 6C), indicating that the ammonia-detoxifying pathway is impaired in the fetal-like hepatocytes. To confirm the reproducibility of these results, we reanalyzed publicly available scRNA-Seq data from hepatocyte spheroids (38) and confirmed that freshly isolated primary hepatocytes strongly express Cps1 and 
A

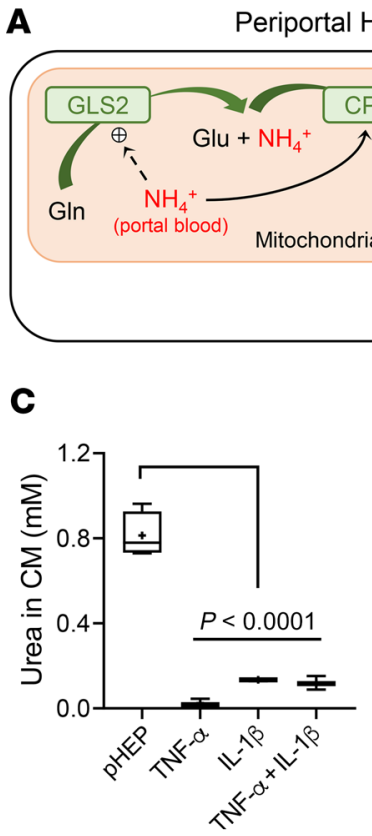

D

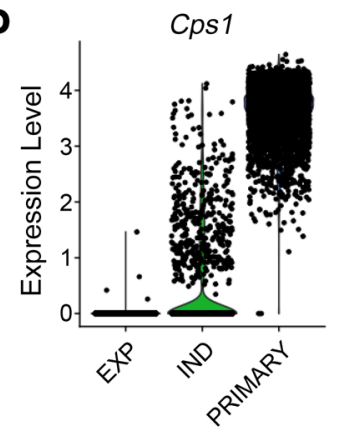

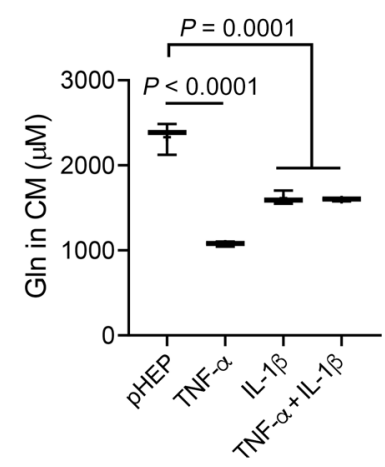

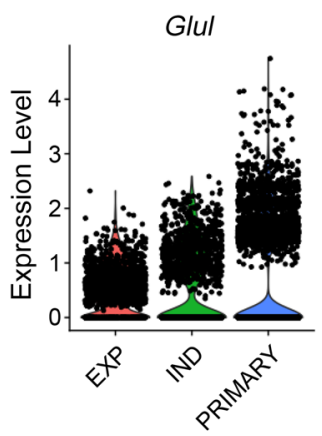

Perivenous HEP

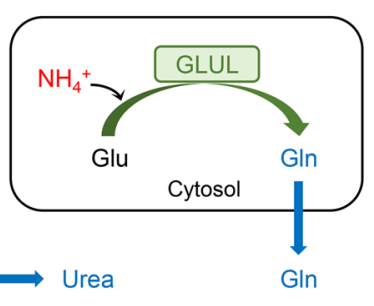

B

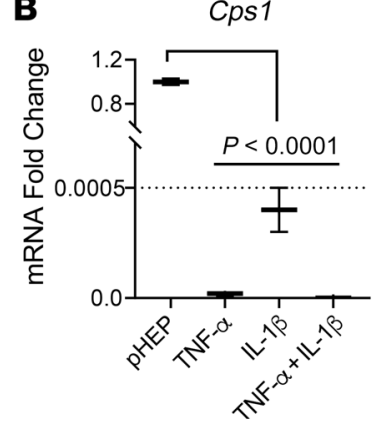

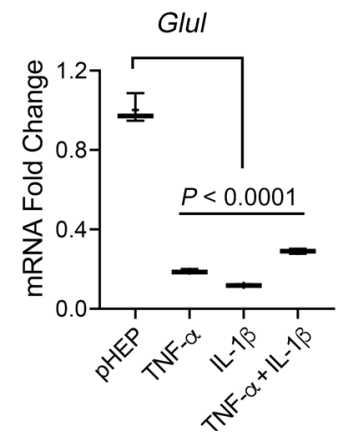

E

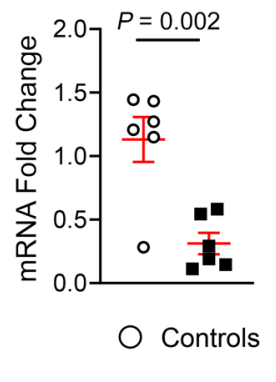

GLUL

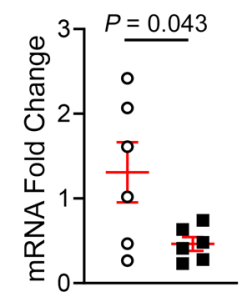

SAH
$\mathbf{F}$

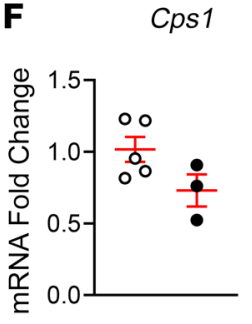

O WT

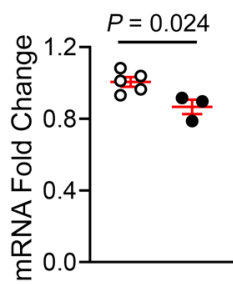

Esrp2-KO
G

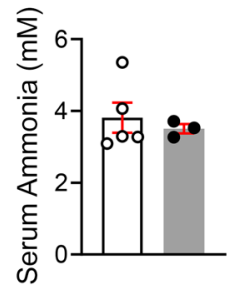

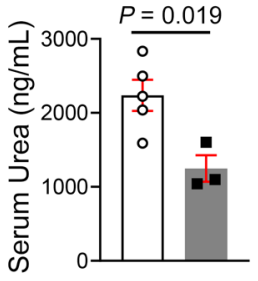

O WT

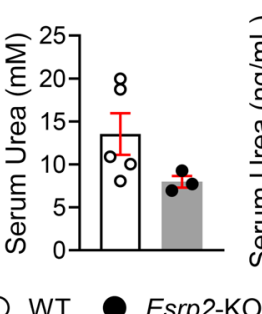

Esrp2-KO

Figure 6. Fetal reprogrammed hepatocytes lose adult liver-specific functions. (A) A schematic of ammonia $\left(\mathrm{NH}_{4}{ }^{+}\right.$) detoxification pathway by hepatocytes in the liver. Glu, glutamate; Gln, glutamine; CP, carbamoyl phosphate. (B) qRT-PCR analysis for Cps1 and Glul in freshly isolated primary mouse hepatocytes and primary hepatocytes cultured under spheroid-forming system for 18 days continuously treated with TNF- $\alpha$, IL-1 $\beta$, or TNF- $\alpha+$ IL-1 $\beta$. (C) The levels of urea, glutamine, and ammonia in conditioned medium of primary hepatocytes as specified. Results are graphed as box and whiskers plots (min to max), and means are indicated as plus signs. Statistical analysis was performed by 1-way ANOVA with Tukey's corrections ( $n=3$ biological replicates/group). (D) scRNA-seq analysis for Cps1 and Glul in primary hepatocytes and hepatocyte spheroids under TNF- $\alpha$-mediated expansion medium or TNF- $\alpha$-withdrawn induction medium, as described in a previous publication (38). (E) qRT-PCR analysis for CPS1 and GLUL in human livers with SAH and healthy controls. Results are graphed as dot plots (SAH, black squares; healthy controls, white circles) showing mean \pm SEM (red bars) ( $n=5$ individuals/group). Statistical analysis was performed by using 2-tailed Student's $t$ test between 2 groups. (F) qRT-PCR analysis for Cps1 and Glul in mouse livers from WT and Esrp2-KO mice at baseline. Results are graphed as dot plots (Esrp2-KO, black circles; WT, white circles) showing mean \pm SEM (red bars) ( $n=3-5$ individuals/group). Statistical analysis was performed by using 2-tailed Student's $t$ test between 2 groups. (G) The serum levels of ammonia, urea, and albumin (ALB) in healthy WT and Esrp2-KO mice. Mean \pm SEM results are graphed, and statistical analysis was performed by using 2-tailed Student's $t$ test between 2 groups ( $n=3-5$ mice/group).

Glul, while both the number of cells expressing these genes and the level of gene expression per cell are reduced when hepatocytes are cultured under TNF- $\alpha$-enriched conditions that promote adult-tofetal reprogramming and spheroid growth (Figure 6D). Further, switching cultures to TNF- $\alpha$-depleted conditions that promote hepatocyte redifferentiation restores both expression of the ammonia detoxification pathway and Esrp2 (Figure 6D), demonstrating that these responses are strictly correlated (Supplemental Figure 
A

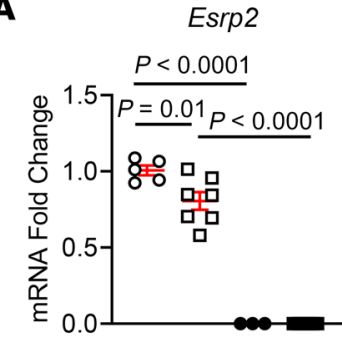

O WT Pair

$\square$ WT 10d+1B

- Esrp2-KO Pair

- Esrp2-KO 10d+1B
Areg

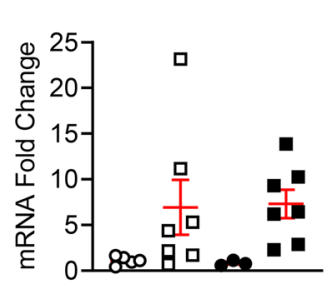

Itgb2

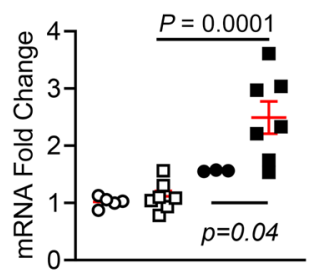

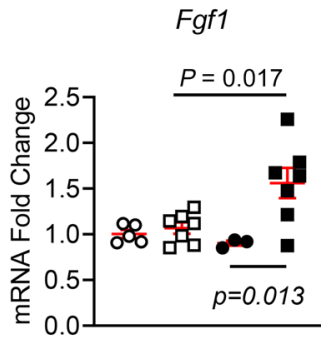

Birc5

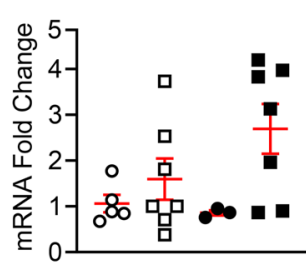

B

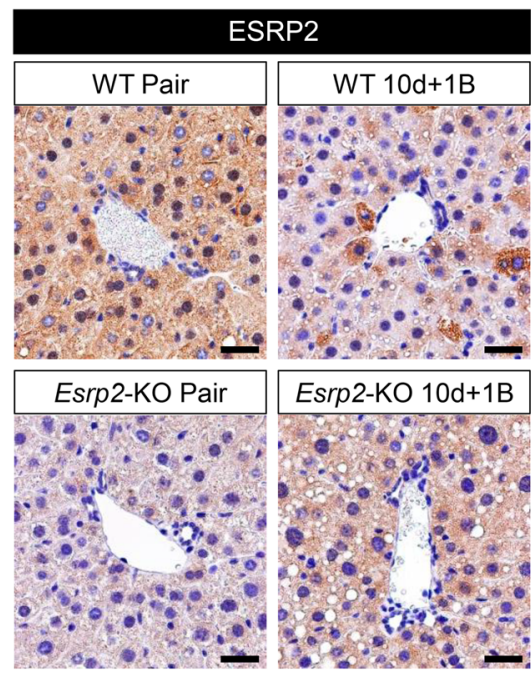

C

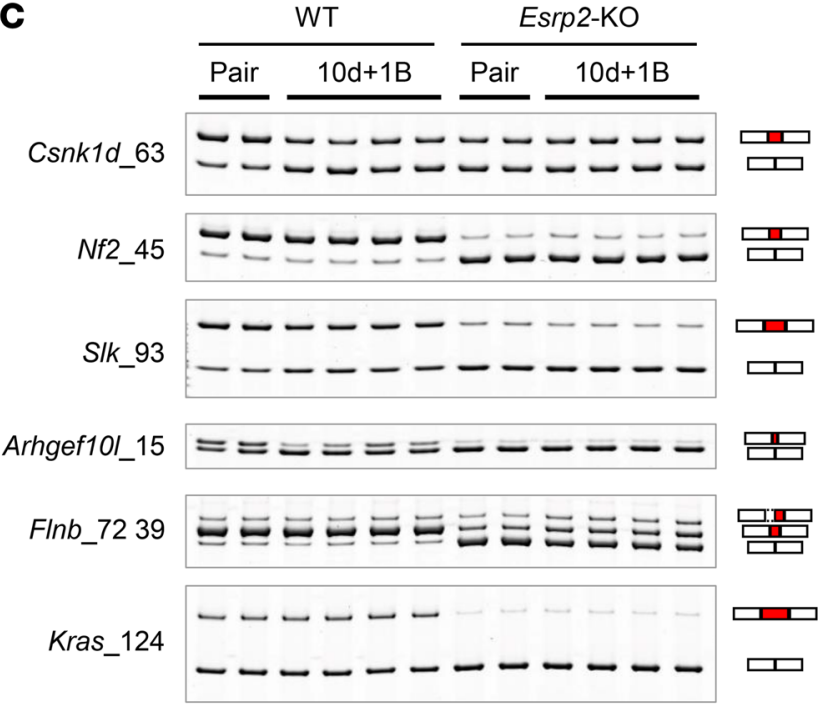

O WT Pair

$\square$ WT 10d+1B

- Esrp2-KO Pair

Esrp2-KO 10d+1B
D
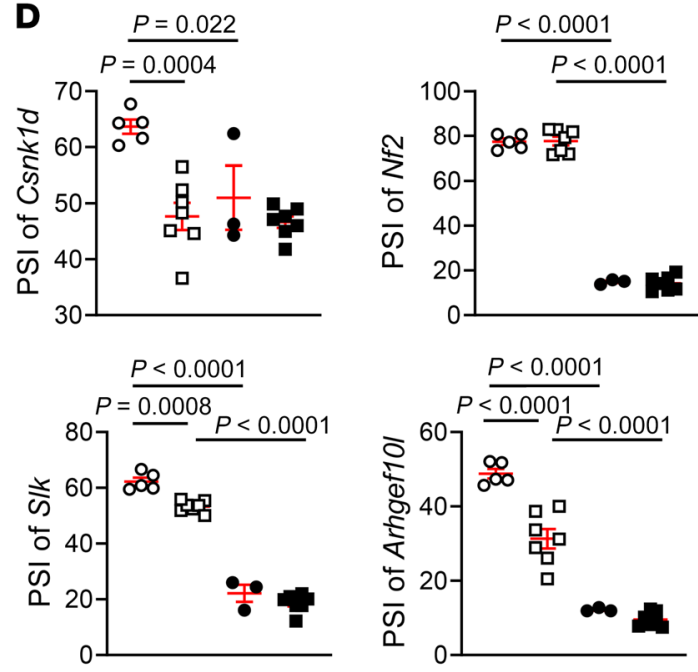

$P<0.0001$

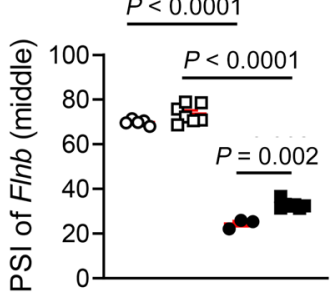

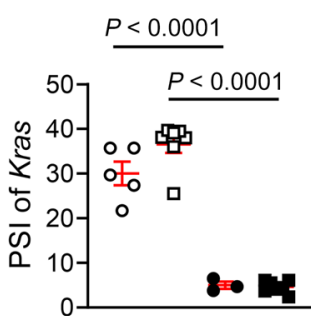

Figure 7. Loss of ESRP2 promotes adult-to-fetal switch in Gao binge model. (A) qRT-PCR analysis for Esrp2 and YAP/TAZ target genes (Areg, Fgf1, Itgb2, and Birc5) in WT and Esrp2-KO mice exposed to 10 days feeding and 1 binge drinking of ethanol (10d + 1B) and pair-fed mice. (B) IHC for ESRP2 in liver sections from these mice. Scale bars: $100 \mu \mathrm{m}$. (C-D) Alternative splicing for HK mRNAs and EMT-related genes (Slk, Arhgef10I, Flnb, and Kras) in WT and Esrp2-KO Gao binge mouse models (C). See full, unedited gels in the supplemental material. All results are graphed as dot plots (WT pair, white circles; WT 10d + 1B, white squares; Esrp2-KO pair, black circles; Esrp2-KO 10d + 1B, black squares) showing mean \pm SEM (red bars) ( $n=3-7$ individuals/group) (D). All statistical analyses were performed by 2-way ANOVA with Tukey's corrections.

6D). Importantly, results from the ex vivo system recapitulate the changes in ammonia-detoxifying genes that occur in human SAH, as evidenced by qRT-PCR data showing that CPS1 and GLUL mRNAs are significantly downregulated in livers of SAH patients compared with healthy controls (Figure 6E). To investigate whether ESRP2 directly regulates ammonia-detoxifying mechanisms, we compared these parameters in uninjured livers of WT and Esrp2KO mice. Hepatic expression of both Cps1 and Glul mRNAs was inhibited markedly by knocking out Esrp2. However, this is insuf- ficient to erode ammonia detoxification because serum levels of ammonia and urea are not significantly different in Esrp2-KO mice versus WT mice (Figure 6, F and G). On the other hand, Esrp2-KO mice had significantly reduced serum levels of albumin, a soluble protein made by the liver, indicating that some adult hepatocyte functions are suppressed in Esrp2-depleted hepatocytes, even in the absence of liver injury (Figure 6G).

Patients with SAH also often exhibit impaired blood clotting. Further, the severity of the coagulopathy is a predictor of short- 

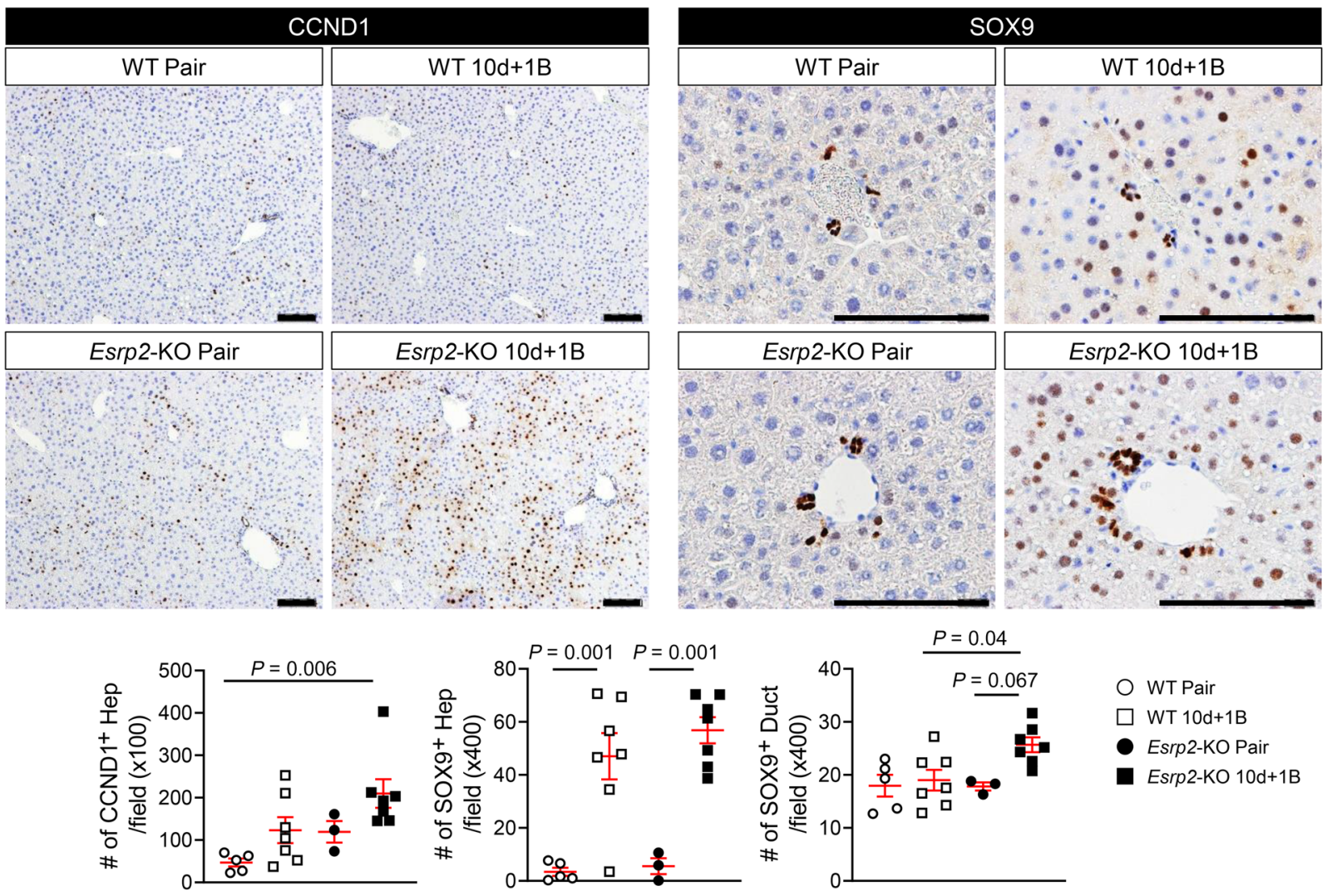

O WT Pair

$\square$ WT 10d+1B

- Esrp2-KO Pair

- Esrp2-KO 10d+1B

Figure 8. ESRP2-depleted hepatocytes become proliferative ductal-like cells after alcohol exposure. IHC for CCND1 and SOX9 in liver sections of WT and Esrp2-KO mice with or without alcohol exposure. The numbers of CCND1+ hepatocytes (Hep) and SOX9+ liver cells (hepatocytes [Hep]; ductal cells [Duct]) were counted in more than 10 randomly selected $\times 100$ or $\times 400$ fields per section, respectively. Scale bars: $100 \mu \mathrm{m}$. Results are graphed as dot plots (WT pair, white circles; WT 10d + 1B, white squares; Esrp2-KO pair, black circles; Esrp2-KO 10d + 1B, black squares) showing mean \pm SEM (red bars) ( $n=3-7$ individuals/group). Statistical analyses were performed by 2-way ANOVA with Tukey's corrections.

term mortality in SAH $(44,45)$. Consistent with this, RNA-Seq data from explanted human SAH livers demonstrated profound suppression of hepatic genes encoding coagulation factors, and the patients with these failing livers had extremely high levels of prothrombin time (PT) and international normalized ratio (INR) (Figure 1B, Supplemental Figure 2, and Supplemental Table 1). To determine whether clotting factor synthesis might also be defective in mouse hepatocytes that have undergone fetal reprogramming, we examined scRNA-Seq data of hepatocyte spheroids and found that fetal-like hepatocytes were also unable to express coagulation factors $(F 2, F 5, F 9, F 10, F 11, F 12, F 13 b)$ and factors that regulate coagulation factors (Proc, Pros1) (Supplemental Figure 7), mimicking the loss of clotting factor gene expression in human $\mathrm{SAH}$ livers. These results provide further evidence that the fetal reprogramming of adult hepatocytes that occurs in SAH has profound functional significance, contributing to the evolution of life-threatening liver failure in patients with SAH.

Adult-to-fetal reprogramming is a common response to tissue injury. To determine whether ESRP2 suppression and adult-to-fetal reprogramming are specific responses to alcohol-induced liver injury or also occur in other types of liver disease, we treated mice with either vehicle (control) or carbon tetrachloride $\left(\mathrm{CCl}_{4}\right)$ for 6 weeks to evoke chronic perivenous (zone 3 ) liver injury and induce progressive liver fibrosis. A progressive decrease in ESRP2 expres- sion occurred in zone 3 hepatocytes that survived $\mathrm{CCl}_{4}$-mediated hepatotoxicity. This was accompanied by progressive accumulation of fetal RNA-splicing variants of the HKs and EMT-related genes (Arhgef1Ol, Slk, Kras) and activation of YAP/TAZ (as evidenced by increased expression of YAP/TAZ target genes, Areg, Birc5) (Supplemental Figure 8, A-C). mRNA expression of liver progenitor markers $I g f 2 b p 3$ and Sox 9 was also substantially greater in livers of $\mathrm{CCl}_{4}$-treated mice than in livers of vehicle-treated controls. Immunostaining for SOX9 confirmed that extensive adult-to-fetal reprogramming occurred in response to $\mathrm{CCl}_{4}$ injury and demonstrated an accompanying accumulation of cyclin D1-positive $\left(\mathrm{CCND}^{+}\right.$) hepatocytes (Supplemental Figure 8, D and E). Repopulation of the liver with these proliferative, relatively immature hepatocytes reduced net expression of several adult hepatocyte genes and was accompanied by decreases in related liver functions. For example, mRNA expression of Glul (which helps to detoxify ammonia by converting it to glutamine) was virtually abolished in $\mathrm{CCl}_{4}$-treated mouse livers (Supplemental Figure $8 \mathrm{~F}$ ) and mRNA levels of Cps1 (which detoxifies ammonia by converting it to urea) tended to be downregulated, although this did not achieve statistical significance (Supplemental Figure 8F). Nevertheless, serum levels of glutamine decreased significantly in the $\mathrm{CCl}_{4}$ model (46) and serum concentrations of urea were significantly lower in $\mathrm{CCl}_{4}$-treated mice than in control mice (Supple- 
A

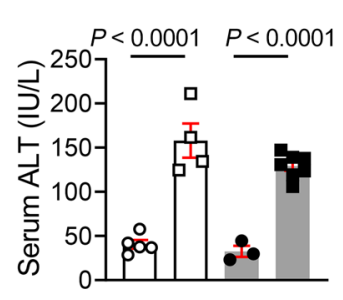

\section{B}

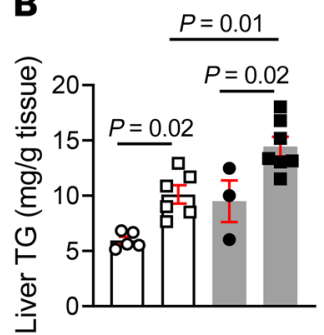

WT Pair Esrp2-KO Pair

$\square$ WT 10d+1B
D

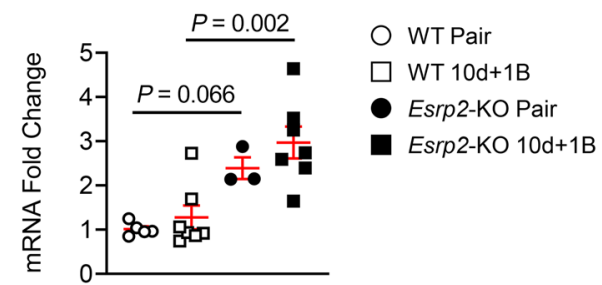

$\mathbf{E}$
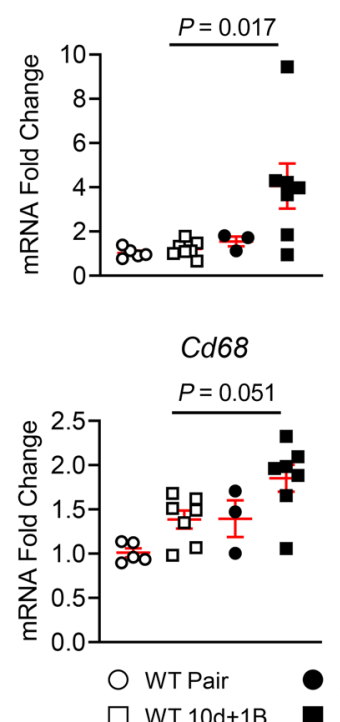

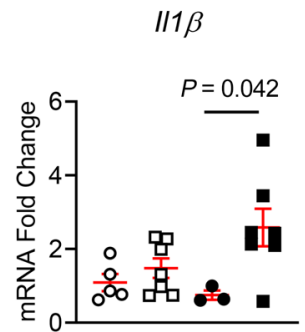

Ly6G

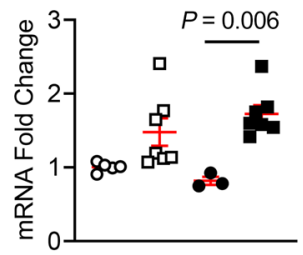

Esrp2-KO Pair

Esrp2-KO 10d+1B
C
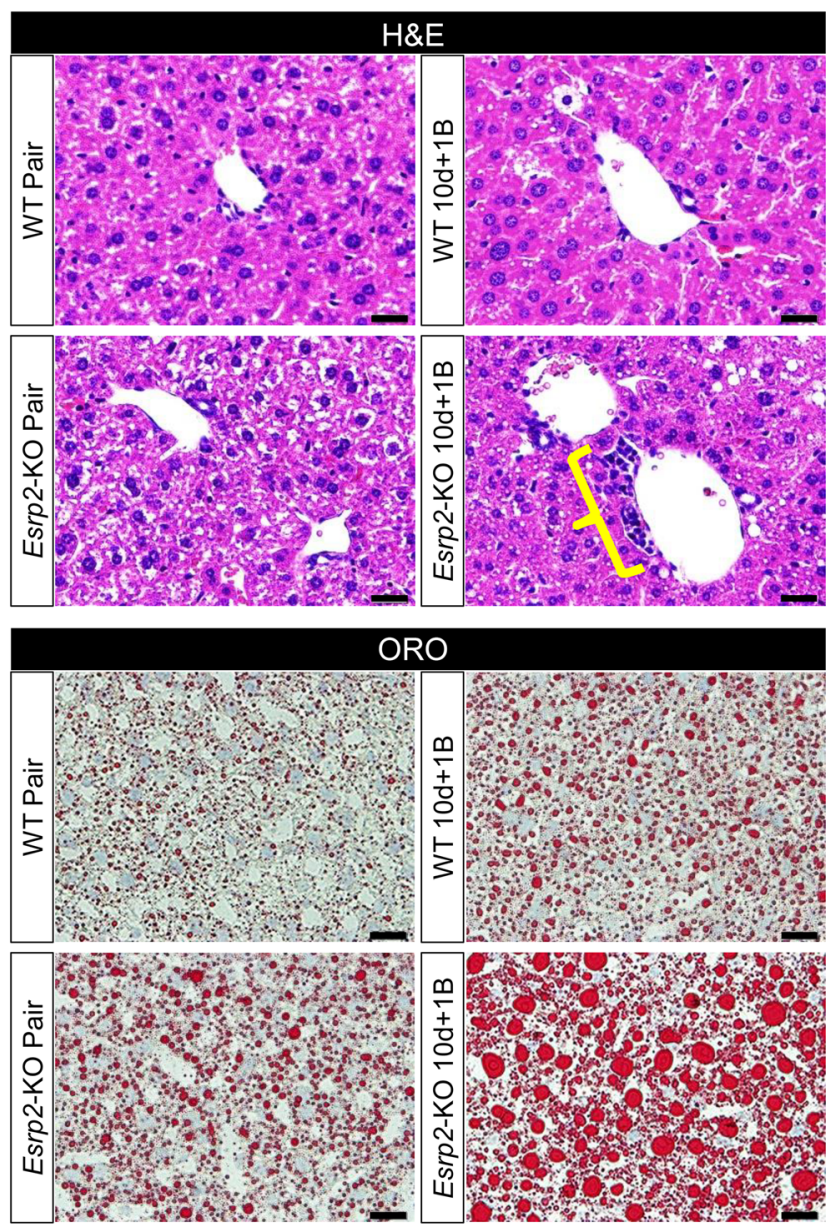

$\mathbf{F}$

Col1 $\alpha 1$

Snail
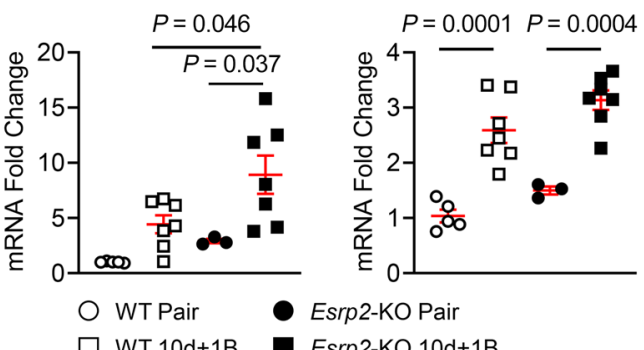

Esrp2-KO Pair

WT 10d+1B Esrp2-KO 10d+1B

Figure 9. Depleting ESRP2 exacerbates alcohol-induced steatohepatitis in mice. (A) The serum levels of ALT in WT and Esrp2-KO mice treated with alcohol as per the Gao binge model. Results are graphed as dot plots (WT pair: white circles with white bar; WT 10d + 1B, white squares with white bar; Esrp2KO pair, black circles with gray bar; Esrp2-KO 10d + 1B, black squares with gray bar) showing mean \pm SEM (red bars) ( $n=3-7$ individuals/group). (B) Hepatic TG content in WT and Esrp2-KO mice with or without alcohol exposure. (C) H\&E and ORO staining in liver sections of these mice. Yellow bracket indicates ductular reaction. Scale bars: $20 \mu \mathrm{m}$. (D-F) qRT-PCR analysis for Ppary (D), inflammatory cytokines (Tnf $\alpha$ and II1 $\beta$ ) and immune cell markers (Cd68 and Ly6C) (E), and fibrogenic markers (Col1 1 and Snail) (F) in WT and Esrp2-KO mice exposed to alcohol or pair-fed control diet. All results are graphed as dot plots (WT pair, white circles; WT 10d + 1B, white squares; Esrp2-KO pair, black circles; Esrp2-KO 10d + 1B, black squares) showing mean \pm SEM (red bars) ( $n$ = 3-7 individuals/group). All statistical analyses were performed by 2-way ANOVA with Tukey's corrections.

mental Figure 8G). Serum levels of total bilirubin were also elevated in $\mathrm{CCl}_{4}$-treated mice, further supporting the concept that some loss of liver-specific functions occurs when persistent suppression of ESRP2 promotes sustained dedifferentiation of hepatocytes in this type of liver injury (Supplemental Figure 8G).

We also determined whether Esrp2 suppression and adult-tofetal reprogramming occur in kidney tissues of mice with severe liver injury caused by $\mathrm{CCl}_{4}$, since inflammatory cytokines that increase in the blood of mice with $\mathrm{CCl}_{4}$-induced liver injury (47) are known to impair renal function in sepsis $(48,49)$ and many etiologies of advanced liver disease, including $\mathrm{SAH}$, in which renal dysfunction is highly predictive of mortality $(4,50)$. Interestingly, we found that Esrp2 tends to be suppressed, the fetal splicing variant of Kras (a direct mRNA target of ESRP2) accumulates, 


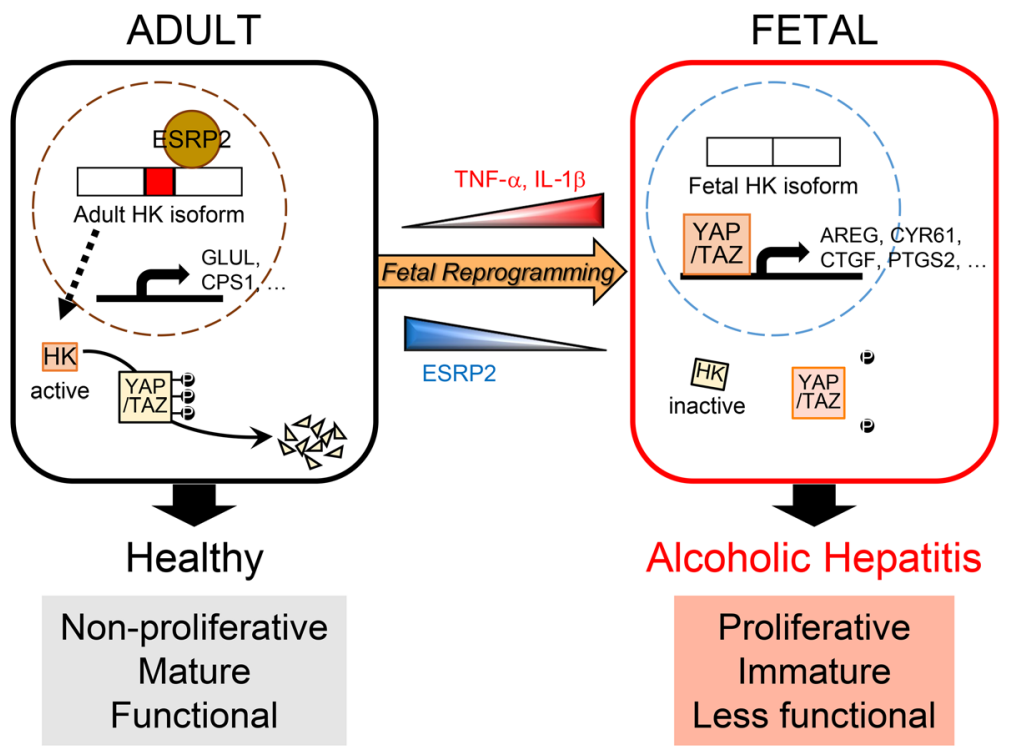

Figure 10. Model for liver failure in severe acute alcoholic hepatitis (SAH). Inflammatory cytokines, such as TNF- $\alpha$ and IL-1 $\beta$, suppress ESRP2, an adult RNA-splicing factor. Loss of ESRP2 permits fetal RNA-splicing variants of HKs to accumulate. Because fetal HKs are relatively inactive, phosphorylation and clearance of their targets, YAP/TAZ, are impaired, allowing these fetal transcription factors to accumulate and reactivate fetal gene expression programs. Thus, the surviving adult hepatocytes are reprogrammed to become more fetal like and regenerative, but less mature. This provokes loss of critical adult hepatocyte functions, including ammonia detoxification and clotting factor biosynthesis, and causes the clinical features of liver failure. and YAP/TAZ are activated, leading to significantly increased expression of YAP/TAZ target genes (Areg, Ptgs2, Birc5) in kidneys of mice with $\mathrm{CCl}_{4}$ liver injury compared with control mice with healthy livers (Supplemental Figure 9, A and B). Considerably more research is required to delineate the role of ESRP2 in the pathogenesis of renal dysfunction associated with severe liver injury, but our provocative initial findings suggest a mechanism that may contribute to the development of hepatorenal syndrome (HRS) in patients with life-threatening SAH (i.e., cytokine-mediated suppression of renal Esrp2 and consequent adult-to-fetal reprogramming of mature renal epithelial cells).

Depleting ESRP2 in adult hepatocytes exacerbates fetal reprogramming in alcohol-injured livers. Evidence linking the loss of Esrp2 to suppression of adult RNA-splicing programs that control vital adult liver functions, such as ammonia detoxification and clotting factor synthesis, suggests that Esrp2 may determine susceptibility to SAH. Therefore, we compared functionally relevant markers of adult-to-fetal reprogramming in WT and Esrp2-KO mice at the end of the Gao binge model of $\mathrm{AH}$, which increases exposure to proinflammatory cytokines, including TNF- $\alpha$ and IL-1 $\beta$ (34). In livers of WT mice with AH, Esrp2 decreased, as expected. Half of the adult splicing events in the 6 ESRP2 targets that we examined were suppressed, including $1 \mathrm{HK}$ mRNA (Csnk1d) and 2 EMT-related genes (Slk, Arhgef10l), and expression of YAP/TAZ target genes (Areg, Birc5) increased slightly but not significantly (Figure 7, A-C). On the other hand, livers of Esrp2KO mice were enriched with fetal splicing variants of all 6 ESRP2 target mRNAs, including both HK mRNAs (Csnk1d, Nf2) and all 4 EMT-related genes (Slk, Arhgef1Ol, Flnb, Kras) (Figure 7C). In addition, YAP/TAZ target genes (Fgf1, Itgb2) were significantly upregulated by the end of the alcohol treatment period (Figure 7A). Thus, in livers of mice with $\mathrm{AH}$, there are dose-dependent relationships among decreased ESRP2, increased fetal RNA-splicing variants, and activation of YAP, a factor that has been proven to stimulate adult hepatocytes to dedifferentiate into proliferative fetal-like progenitor cells that coexpress hepatocyte and ductal cell markers. Therefore, we determined whether the hepatocytes of Esrp2-
KO mice are more proliferative and fetal-like than WT hepatocytes after alcohol-induced liver injury. IHC for CCND1 demonstrated rare $\mathrm{CCND} 1^{+}$hepatocytes in WT mice before alcohol feeding and showed that proliferative hepatocytes increased in WT livers with AH. As expected (12), Esrp2-KO hepatocytes were more proliferative than WT hepatocytes at baseline. Further, livers of Esrp2-KO mice became massively repopulated by proliferative hepatocytes after alcohol exposure (Figure 8). Immature hepatocytes expressing the ductal marker SOX9 were barely detected in either WT or Esrp2-KO mice before alcohol feeding, although mature-appearing bile duct cells themselves strongly expressed SOX9 in both groups (Figure 8). After alcohol exposure, both WT and Esrp2-KO mice accumulated $\mathrm{SOX} 9^{+}$hepatocytes and a SOX $9^{+}$ductular-type reaction emerged in Esrp2-KO mice (Figure 8). Hence, the aggregate data suggest that ESRP2-depleted hepatocytes are primed to be reprogrammed into proliferative fetal-like cells and confirm that loss of ESRP2 exacerbates fetal reprogramming induced by alcohol, supporting the tenet that ESRP2 mediates susceptibility to liver dysfunction related to $\mathrm{AH}$. To verify that these responses result from direct effects of ESRP2 suppression in hepatocytes, we silenced Esrp2 in AML12 cells and confirmed that directly inhibiting ESRP2 in hepatocytes increased fetal splicing variants, upregulated expression of multiple YAP/TAZ target genes (Areg, Cyr61, Ctgf, Fgf1, Itgb2), and induced various markers of proliferative progenitor cells (Ccnd1, Ck7, Snail, Vim, EpCAM) (Supplemental Figure 10, A-D).

Depleting ESRP2 exacerbates alcohol-induced steatohepatitis in mice. Depleting ESRP2 clearly enhances susceptibility to injury-related dedifferentiation of hepatocytes and this compromises liver function. However, whether repopulating the liver with immature cells influences other outcomes of liver injury remains uncertain. Therefore, we treated Esrp2-KO mice and WT mice with alcohol according to the Gao binge model of AH and compared liver injury, steatosis, inflammation, and fibrosis. Alcohol treatment increased serum levels of alanine transaminase (ALT) comparably in WT and Esrp2-KO mice, suggesting that the severity of alcohol-induced liver injury is not determined by 
Esrp2 (Figure 9A). Hepatic steatosis also increased significantly after alcohol treatment in both groups of mice, as assessed by hepatic triglyceride (TG) quantification and oil red O (ORO) staining (Figure 9, B and C). However, liver TG content and ORO staining were greater in Esrp2-KO mice than in WT mice, and we found that Esrp2-depleted livers expressed higher mRNA levels of Ppary (Figure 9D), a transcription factor that induces lipid accumulation in the liver (51), suggesting that depleting Esrp2KO enhances susceptibility to hepatic steatosis. As mentioned earlier, Esrp2-KO mice also developed a more robust ductular reaction than WT mice after chronic alcohol consumption (Figure 8 and Figure 9C). The intensities of the ductular reaction, liver inflammation, and liver fibrogenesis typically correlate with each other (52) because immature liver cells themselves produce chemokines as well as profibrogenic and proinflammatory factors to promote wound-healing responses in damaged livers $(5,7)$. Consistent with these data, expression of genes encoding inflammatory cytokines $(\operatorname{Tn} f \alpha, I l 1 \beta)$ and markers of macrophages $(C d 68)$ and neutrophils $(L y 6 G)$ were upregulated in the livers of alcohol-fed Esrp2-KO mice, while none of these genes were significantly induced in the livers of WT mice after alcohol feeding (Figure 9E). mRNA levels of genes encoding fibrosis markers (Col1 $\alpha 1$, Snail) were also significantly upregulated in alcohol-injured livers of Esrp2-KO mice (Figure 9F), although the Gao binge model of $\mathrm{AH}$ causes relatively mild injury and no detectable liver fibrosis (35). Together, the findings in mice confirm that alcohol-induced liver injury promotes suppression of ESRP2 and support the concept that the intensity of the resultant wound-healing response parallels the level of ESRP2 suppression, adult-to-fetal reprogramming, and liver repopulation by immature liver cells. Taken together, our results reveal a mechanism that may explain why acute liver failure sometimes develops in individuals who drink alcohol excessively and identify TNF- $\alpha$, IL-1 $\beta$, and ESRP2 as critical regulators of that process (Figure 10).

\section{Discussion}

One-month mortality rates in patients hospitalized with SAH are higher than those reported for many other potentially fatal diseases, including myocardial infarction, stroke, and most cancers (53, $54)$. Death results from progressive and global loss of normal liver functions in SAH, as evidenced by the fact that early replacement of the damaged liver restores both acute and long-term survival (55). Unfortunately, liver transplantation is not an option for most $\mathrm{SAH}$ patients, and thus, effective medical therapies are desperately needed. However, developing a successful treatment for $\mathrm{SAH}$ has proven challenging due to uncertainties regarding the pathogenesis of SAH-related acute liver failure. In this study, we demonstrate that lethal liver failure in SAH is caused by massive accumulation of immature hepatocytes and identify mechanisms that critically control that process, thereby revealing diagnostic and therapeutic targets for this deadly disease. Based on these findings, we also propose a model for the pathogenesis of acute liver failure in SAH whereby TNF- $\alpha$ and IL-1 $\beta$, inflammatory cytokines that are increased by excessive alcohol ingestion, massively suppress ESRP2, an adult RNA-splicing factor that maintains the nonproliferative, mature phenotype of adult hepatocytes (Figure
10). The loss of ESRP2 function permits reemergence of a fetal RNA-splicing program that enables hepatocytes that survive alcohol injury to shed adult hepatocyte functions in order to become more regenerative and heal alcohol-related liver damage. However, vital liver functions are dangerously eroded, and overall survival is threatened when this regenerative response is so exuberant that the liver becomes excessively populated by functionally immature hepatocytes.

Multiple lines of evidence support this proposed model. Most importantly, mRNA and protein levels of ESRP2, an RBP that is selectively expressed in mature hepatocytes, are drastically reduced in explanted livers of patients who required urgent liver transplantation to survive SAH. ESRP2 expression and activity are also decreased in several mouse models of $\mathrm{AH}$, and the level of ESRP2 suppression correlates with the intensity of alcohol exposure and severity of liver inflammation, supporting the tenet that alcohol-inducible proinflammatory cytokines downregulate ESRP2. Studies in cultured adult hepatocytes confirm these findings, as TNF- $\alpha$ and IL-1 $\beta$ directly inhibit Esrp2. Further research is needed to evaluate the effects of other cytokines on hepatocyte reprogramming.

Critically pertinent to the pathogenesis of liver failure, reducing ESRP2 in hepatocytes has profound functional consequences. In human SAH livers that are massively populated by ESRP2-depleted hepatocytes, key adult RNA-splicing programs are replaced by fetal programs, the hepatic transcriptome demonstrates global loss of mature liver functions, and signaling pathways that promote EMT and growth of immature cells are induced. Similar responses accompany ESRP2 suppression in the mouse AH models. These adult-to-fetal phenotypic "switches" are direct consequences of ESRP2 inhibition because they are recapitulated in cultured adult hepatocytes by knocking down Esrp2 with siRNA or treating the cells with TNF- $\alpha / \mathrm{IL}-1 \beta$ to suppress ESRP2 expression. Further, the dedifferentiation process is reversed by reintroducing Esrp2. It is particularly noteworthy that suppressing ESRP2 in cultured hepatocytes directly inhibits their expression of clotting factors and blocks ammonia detoxification, given that coagulopathy and hyperammonemia are potentially lethal consequences of liver failure and predict acute mortality in SAH $(39,56)$.

Previous and ongoing research suggest that suppression of ESRP2 is a conserved response to liver injury. For example, we reported that after $\mathrm{PH}$, subpopulations of regenerating mouse hepatocytes transiently suppress their expression of ESRP2 and mature hepatocyte markers (e.g., $\mathrm{C} / \mathrm{EBP} \alpha$ ) but acquire markers of fetal-like cells (e.g., IGF2BP3, YAP, and SOX9) (16). That paper also describes striking accumulation of ESRP2- hepatocytes in explanted livers from patients with drug-induced acute liver failure. In addition, others recently reported RNA-Seq data that demonstrate that Esrp2 transcripts are significantly decreased in patients with nonalcoholic steatohepatitis compared with control subjects without known liver disease (57). Here, we show that hepatic expression of both Esrp2 mRNA and ESRP2 protein is inhibited in a mouse model of toxin-induced liver injury. In aggregate, these data indicate that accumulation of ESRP2- hepatocytes and the extent of hepatocyte dedifferentiation generally parallel the intensity and duration of liver injury and predict the severity of liver dysfunction. 
ESRP2 expression is not strictly restricted to liver epithelia $(58,59)$. Thus, our evidence that ESRP2 expression is directly suppressed by TNF- $\alpha$ and IL- $1 \beta$ may also have implications for the pathogenesis of extrahepatic organ failure in patients with liver injury, given that circulating levels of inflammatory cytokines as well as lipopolysaccharide and other cytokine-inducing factors increase in many liver diseases $(50,60)$. Alcohol consumption per se can also increase systemic exposure to gut-derived inflammatory signals by disrupting the intestinal epithelial barrier (61). Similarly, sepsis provokes systemic inflammation even in the absence of liver injury (62). Further, our results show that tissues that become repopulated by ESRP2-deficient, fetal-like cells develop a microenvironment that promotes local inflammation and fibrogenesis. Our findings in kidneys from mice with chronic $\mathrm{CCl}_{4}$-induced liver injury support the tenet that cytokinemediated adult-to-fetal epithelial reprogramming may generally be involved in inflammation-related organ dysfunction and justify further research to assess the potential role of ESRP2 in the pathogenesis of multiple organ failure. The results will be particularly pertinent for SAH patients because $\mathrm{SAH}$ is known to increase the risk for developing HRS, progressive kidney failure, and high short-term mortality (63-65). Moreover, increased levels of circulating cytokines, including TNF- $\alpha$ and IL-1 $\beta$, have already been implicated as biomarkers for susceptibility to HRS in SAH patients (65-67) and therapies that inhibit evolution of HRS have been reported to improve survival in SAH $(50,68)$.

Evidence that ESRP2 suppression promotes SAH mortality fundamentally shifts current paradigms for liver failure pathogenesis because the loss of ESRP2 enhances, rather than inhibits, the viability and regenerative capabilities of adult hepatocytes. Thus, contrary to the conventional dogma, livers do not fail in patients with fatal SAH because inflammatory cytokines are cytotoxic. Instead, these livers fail because inflammatory cytokines (e.g., TNF- $\alpha$ and IL-1 $\beta$ ) disrupt molecular signaling programs that normally constrain dedifferentiation of adult hepatocytes, causing functional parenchyma to be replaced by the fetal-like cells that are lacking liver-specific functions. In this regard, failing $\mathrm{SAH}$ livers functionally resemble livers where normal parenchyma is replaced by malignant cells, except that the fetal-like hepatocytes in SAH can reacquire their mature phenotype after the factors that caused them to dedifferentiate dissipate. This helps to explain how it is possible to survive SAH and why conditions that exacerbate cytokine exposure (e.g., infections and recurrent alcohol abuse) increase SAH mortality $(37,60)$. Paradoxically, despite all the evidence indicating an important role for ESRP2 suppression in cytokine-mediated liver failure, ESRP2 is completely dispensable for survival in the absence of liver injury because Esrp2-KO mice do not develop liver failure spontaneously. Further, Esrp2 is suppressed transiently when the liver regenerates after PH (16) and overexpressing ESRP2 in hepatocytes blocks regeneration and causes progressive liver damage after injury (12). The latter observations may explain why abrogating TNF- $\alpha$ signaling also proved to be deadly in SAH $(69,70)$.

In summary, we have identified a previously unexpected role for an RBP, ESRP2, in the pathogenesis of SAH-related liver failure, demonstrated how ESRP2-dependent RNA-splicing programs critically influence liver functioning after alcohol injury, and shown that this process is directly regulated by TNF- $\alpha$ and IL-1 $\beta$, inflammatory cytokines that mediate SAH pathogenesis. These findings reveal tractable targets that might be modulated to optimize recovery from SAH while emphasizing the need for further research to map the signaling networks that regulate hepatocyte plasticity in adulthood.

\section{Methods}

Human subjects. This study evaluated 6 healthy donor liver tissues and 6 liver tissues from SAH patients who were referred for liver transplantation at Johns Hopkins University Hospital.

RNA-Seq analysis. RNA-Seq analysis was performed using high-quality RNA samples from 5 patients with SAH and 5 healthy controls as described. All original microarray data were deposited in the NCBI's Gene Expression Omnibus database (GEO GSE143318). Genes were considered as having significant differential expression following imposed cutoff clearance $\left(\mathrm{FDR}<0.05, \mid \log _{2}\right.$ [fold change] $>0.6)$. KEGG pathway analysis and annotation of gene tissue expression were performed using DAVID (version 6.8) $(71,72)$ and mapped using Enrichment Maps plugin in Cytoscape (version 3.7.1) (73).

Animal studies. Male WT C57BL/6J (Jackson Laboratory) and Esrp2-KO mice (University of Illinois at Urbana-Champaign), generated as previously described (13), were fed with short-term chronic (10 days) and 1 binge ethanol (the NIAAA or Gao binge model), as described in Bertola et al. (35). Briefly, both WT $(n=12)$ and Esrp2KO mice $(n=10)$ were initially fed the control Lieber-DeCarli diet ad libitum for 5 days to acclimatize them to the liquid diet. Subsequently, ethanol-fed groups were allowed free access for 10 days to the ethanol diet containing $5 \%(\mathrm{v} / \mathrm{v})$ ethanol, and control groups were pair-fed with the isocaloric control diet. At day 11, ethanol-fed and pair-fed mice received a single dose of ethanol ( $5 \mathrm{~g} / \mathrm{kg}$ body weight) or isocaloric maltose dextrin, respectively, via gavage in the early morning and were sacrificed 9 hours later. The HF liquid diet \pm ethyl alcohol (EtOH) model was also generated as described previously $(5,74)$. Briefly, male WT mice (Jackson Laboratory) were fed HF diet with or without EtOH via continuous intragastric infusion for 4 weeks. The liver tissues were collected for subsequent analyses.

Frozen mouse liver tissues from the Tsukamoto-French model or DDC $\pm \mathrm{EtOH}$ model were provided in-house. Briefly, the Tsukamoto-French model was generated by permitting mice to consume a HF/high-cholesterol Western diet ad libitum for 2 weeks, followed by intragastric infusion of EtOH liquid diet $(27 \mathrm{~g} / \mathrm{kg} / \mathrm{d})$ for 8 weeks with weekly EtOH binges $(4-5 \mathrm{~g} / \mathrm{kg})$ started from the second week of intragastric feeding. Control mice were administered isocaloric diet without EtOH and gavaged with dextrin maltose (75). The DDC \pm EtOH model was generated by feeding male WT C57BL/6J mice with $0.05 \%$ of the DDC diet or base diet for 8 weeks. Animals then underwent surgical intragastric intubation (36). After surgery, mice were housed in individual metabolic cages and allowed 2 weeks to recover with ad libitum access to food and water. Afterward, to subgroups of DDC diet-fed mice, either control HF diet or EtOH was delivered continuously through the intragastric cannula up to at $24 \mathrm{~g} / \mathrm{kg} / \mathrm{d}$ by gradually increasing doses for 4 weeks.

To generate a type of severe nonalcoholic liver injury, C57BL/6 mice were treated with $0.6 \mathrm{~mL} / \mathrm{kg} \mathrm{CCl}_{4}(n=7$ mice) or corn oil ( $n=4$ mice) as vehicle by intraperitoneal injection twice a week for 6 weeks. Liver and kidney tissues and blood samples were collected for subsequent analyses. 
qRT-PCR and splice isoform analysis. Total RNAs were isolated from whole liver tissues or cell lysates using AllPrep DNA/RNA/miRNA Universal Kit (QIAGEN) or TRIzol reagent (Invitrogen). The concentration and purity of RNA were determined using NanoDrop 2000 (Thermo Fisher Scientific). Template complementary DNAs were synthesized from total RNA using the High-Capacity cDNA Reverse Transcription Kit (Applied Biosystems) according to the manufacturer's protocols. Platinum II Hot-Start Green PCR Master Mix (Invitrogen) was used for PCR-based alternative splicing assays, and Power SYBR Green Master Mix (Applied Biosystems) was used for real-time qPCR according to the manufacturer's specifications (StepOnePlus RealTime PCR System, Applied Biosystems). PSI values for the variably spliced regions were calculated with CS Analyzer 4 software (ATTO Corp.) as follows: ([exon inclusion band intensity]/[exon inclusion band intensity + exon exclusion band intensity] $\times 100$ ). qRT-PCR results were normalized to the housekeeping gene $40 \mathrm{~S}$ ribosomal proteins S9 (RPS9) mRNA based on the threshold cycle $\left(C_{t}\right)$, and relative fold-change was determined using the $2^{-\Delta \Delta \mathrm{Ct}}$ method. The sequences of primers used in this study are listed in Supplemental Table 2.

Cultures of mouse hepatocyte cell line. AML12 cells (ATCC) were cultured in DMEM/F12 Medium (Gibco, Thermo Fisher Scientific) supplemented with $10 \%$ FBS (Gibco, Thermo Fisher Scientific), 1× premixed insulin-transferrin-selenium (Gibco, Thermo Fisher Scientific), $40 \mathrm{ng} / \mathrm{mL}$ dexamethasone, and 1× gentamycin (Gibco, Thermo Fisher Scientific) at $37^{\circ} \mathrm{C}$ in a humidified atmosphere containing $5 \% \mathrm{CO}_{2}$. Cells were serum starved for 16 hours before treatment. After serum starvation, cells were released in reduced serum (1\% FBS) for up to 48 hours. For knockdown experiments, ON-TARGETplus SMARTpool siRNA for mouse Esrp2 (GE Healthcare Dharmacon Inc.) or ON-TARGETplus Non-targeting Control Pool (Dharmacon) was purchased. AML12 cells were transfected with gene-specific siRNA oligos (20 $\mathrm{nM}$ ) using Lipofectamine RNAiMAX (Invitrogen), and 48 hours after transfection, cells were lysed for RNA and protein assays. For TNF- $\alpha$ and IL-1 $\beta$ treatment, AML12 cells were cultured with or without recombinant murine TNF- $\alpha$ (100 ng/mL, Peprotech), IL-1 $\beta$ (100 ng/mL, Peprotech), or a combination of TNF- $\alpha$ and IL-1 $\beta$ ( $50 \mathrm{ng} / \mathrm{mL}$ each, total $100 \mathrm{ng} / \mathrm{mL}$ ) and harvested after 24 hours for subsequent analyses. Cell proliferation assay was performed using commercial Cell Counting Kit-8 (CCK8) (Dojindo Molecular Technologies Inc.).

Cocultures between AML12 cells and RAW264.7 cells (mouse macrophage cell line from ATCC) were conducted using Transwell inserts (Corning Inc.) in which culture medium was diffusible, but cells were not permeable. RAW264.7 cells were added into Transwell inserts above monolayers of AML12 cells, and they were cocultured for 24 hours. Afterward, AML12 cells were lysed for subsequent analysis and the levels of TNF- $\alpha$ in conditioned medium were evaluated with a commercial ELISA kit for mouse TNF- $\alpha$ (Invitrogen) according to the manufacturer's instructions.

Spheroid culture of primary mouse hepatocytes. Primary mouse hepatocytes were isolated from healthy livers of WT male mice using a 2-step collagenase perfusion technique as described previously (16). Briefly, mice were anesthetized with isoflurane to immobilize them in the recumbent position on a treatment table, the inferior vena cava was cannulated under aseptic conditions, and the portal vein was cut. Livers were perfused in situ with EGTA at $37^{\circ} \mathrm{C}$ and then collagenase type IV (Roche) in PBS (Gibco, Thermo Fisher Scientific). After the liver was digested, it was dissected out, cut into small pieces, and passed through a $100 \mu \mathrm{m}$ strainer. Hepatocytes were separated from nonparenchymal cells by gravity sedimentation. The purity of the isolated hepatocytes was evaluated with an assessment of the relative percentage of hepatocytic-appearing cells using standard microscopy; this is typically over $96 \%$ regardless of the timing of hepatocyte isolation.

For the 3D culture of primary mouse hepatocytes, we followed the protocols previously reported by Peng et al. (38). Primary mouse hepatocytes were embedded in growth factor reduced Matrigel (BD) at approximately 20,000 cells per well in 24-well plates. To expand hepatocyte spheroids, $3 \mu \mathrm{M}$ CHIR99021 (Peprotech), 25 ng/mL EGF (Peprotech), and $50 \mathrm{ng} / \mathrm{mL}$ HGF (Peprotech) were added to the basal medium described as follows: William's E medium containing 1\% (v/v) Glutamax (Gibco, Thermo Fisher Scientific), 1\% (v/v) nonessential amino acids (Gibco, Thermo Fisher Scientific), 1\% (v/v) penicillin/ streptomycin (Gibco, Thermo Fisher Scientific), 0.2\% (v/v) normocin (Invitrogen), 2\% (v/v) B27 (Gibco, Thermo Fisher Scientific), 1\% (v/v) N2 supplement (Gibco, Thermo Fisher Scientific), $10 \mathrm{mM}$ nicotinamide (Sigma-Aldrich), $1.25 \mathrm{mM} \mathrm{N}$-acetylcysteine (Sigma-Aldrich), $10 \mu \mathrm{M}$ Y27632 (Peprotech), and $1 \mu \mathrm{M}$ A83-01 (Tocris Bioscience). To examine the effect of TNF- $\alpha$ and IL-1 $\beta$ on hepatocyte expansion, 100 $\mathrm{ng} / \mathrm{mL}$ TNF- $\alpha$ or IL-1 $\beta$ (Peprotech) was also included in the expansion medium. In some experiments, adenoviruses expressing mouse ESRP2 or GFP as a control were transduced into hepatocyte spheroids under TNF- $\alpha$-mediated expansion conditions. Medium was replaced every 2 to 3 days.

For qRT-PCR and splice isoform analysis from the hepatocyte spheroids, spheroids were harvested from Matrigel using dispase (Stem Cell Technologies) and total RNA was extracted using the RNeasy Mini Kit (QIAGEN) according to the manufacturer's instructions. The steps following were the same as described above.

For whole mount immunofluorescence staining, hepatocyte spheroids were processed and stained as described by others (76). Briefly, culture medium was aspirated, and hepatocyte spheroids were fixed with $10 \%$ formalin and permeabilized after growth for 14 days. Fixed spheroids were blocked in normal goat serum according to the species in which secondary antibody was raised, followed by overnight incubation with primary antibodies (rabbit anti-YAP or rabbit antiTAZ that were used for IHC) at $4^{\circ} \mathrm{C}$. At day 2 , spheroids were washed 3 times and incubated with secondary antibody conjugated with red fluorescein (goat anti-rabbit IgG Alexa Fluor 647, Invitrogen) for 2 hours. After several washes, 1 droplet of VECTASHIELD Antifade Mounting Medium with DAPI (Vector Laboratories Inc.) was added and images were visualized using fluorescence microscope.

Single-cell RNA-Seq analysis. Single-cell RNA-Seq was performed using the 10× Genomics Chromium System. The Cell Ranger Software (version 2.1.1) was used to perform sample demultiplexing, barcode processing, and 3' UMI counting. For primary hepatocytes, we removed cells with fewer than 200 expressed genes and fewer than 1800 UMIs as well as cells with fractions of mitochondrial UMIs (>20\%). Approximately 4500 cells were sequenced. For expansion culture and induction culture, data sets derived from 1 male and 1 female were obtained from the GEO database (GSE120067); this was previously described here (38). Data was reprocessed using Cell Ranger software with mm10 as the reference genome. Single-cell data were integrated and analyzed using the workflow from the Seurat R Package (77). The top 10 principal components by PCA analysis were selected for downstream clustering analysis. UMAP plots were used to visualize the clusters. 
Statistics. Data are expressed as mean \pm SEM unless otherwise specified. Statistical significance between 2 groups was analyzed by 2-tailed Student's $t$ test, whereas comparisons of multiple groups were evaluated by 1-way or 2-way ANOVA as specified followed by a post hoc Tukey's test. Correlations were assessed by Pearson's correlation coefficient $(r) . P<0.05$ was considered statistically significant. Drawing graphs and statistical analyses were performed using GraphPad Prism 8 (GraphPad Software Inc.).

For further information, see Supplemental Methods.

Study approval. The human research was approved by the Human Ethics Committee of Johns Hopkins University Hospital (IRB 00107893), and written, informed consent was obtained from all participants. Human samples were used in accordance with the NIH and institutional guidelines for human subject research. Animal studies were conducted under approved Duke University (A262-18-11) and University of Illinois at Urbana-Champaign Institutional Animal Care and Use Committee (IACUC) protocols as per the Guide for the Care and Use of Laboratory Animals (National Academies Press, 2011).

\section{Author contributions}

$\mathrm{JH}$ designed the research studies, conducted experiments, acquired data, analyzed data, provided reagents, and wrote and revised the manuscript. ZS and ARA provided human liver sam- ples and RNA-Seq data. SB, KD, UVC, and TC conducted experiments and acquired data. HT and IR provided mouse liver samples. AK provided reagents and Esrp2-KO mice, conducted some of the alcohol feeding studies, and provided funding to support some of the alcohol feeding studies. AMD designed the research studies, analyzed data, wrote and revised the manuscript, and secured funding for the research.

\section{Acknowledgments}

This work was supported by NIH grants DK106633, AA010154, DK077794 (to AMD), HL126845 (to AK), and R24 AA025017 (to ZS); the Florence McAlister Professorship of Medicine (to AMD); a Planning Grant Award from Cancer Center at Illinois (to AK); a postdoctoral fellowship from the Duke Regeneration Next Initiative (to JH); the NIH Tissue microenvironment training program T32-EB019944 (to SB); and the Animal Core of the NIAAA Alcohol Research Center at USC (to HT).

Address correspondence to: Anna Mae Diehl, 905 South LaSalle Street, GSRB I, Suite 1073, Durham, North Carolina 27710, USA. Phone: 919.684.4173; Email: annamae.diehl@duke.edu. Or to: Auinash Kalsotra, University of Illinois, 461 Medical Sciences Building, 506 South Mathews Avenue, Urbana, Illinois, USA. Phone: 217.300.7654; Email: kalsotra@illinois.edu.
1. Louvet A, Mathurin P. Alcoholic liver disease: mechanisms of injury and targeted treatment. Nat Rev Gastroenterol Hepatol. 2015;12(4):231-242.

2. Yu CH, Xu CF, Ye H, Li L, Li YM. Early mortality of alcoholic hepatitis: a review of data from placebo-controlled clinical trials. World J Gastroenterol. 2010;16(19):2435-2439.

3. Orntoft NW, Sandahl TD, Jepsen P, Vilstrup H. Short-term and long-term causes of death in patients with alcoholic hepatitis in Denmark. Clin Gastroenterol Hepatol. 2014;12(10):1739-44.e1.

4. Altamirano J, et al. Acute kidney injury is an early predictor of mortality for patients with alcoholic hepatitis. Clin Gastroenterol Hepatol. 2012;10(1):65-71.e3.

5. Jung Y, et al. Accumulation of hedgehog-responsive progenitors parallels alcoholic liver disease severity in mice and humans. Gastroenterology. 2008;134(5):1532-1543.

6. Sancho-Bru P, et al. Liver progenitor cell markers correlate with liver damage and predict shortterm mortality in patients with alcoholic hepatitis. Hepatology. 2012;55(6):1931-1941.

7. Aguilar-Bravo B, et al. Ductular reaction cells display an inflammatory profile and recruit neutrophils in alcoholic hepatitis. Hepatology. 2019;69(5):2180-2195.

8. Achanta S, Verma A, Srivastava A, Nilakantan H, Hoek JB, Vadigepalli R. Single-cell gene expression analysis identifies chronic alcohol-mediated shift in hepatocyte molecular states after partial hepatectomy. Gene Expr. 2019;19(2):97-119.

9. Yimlamai D, et al. Hippo pathway activity influences liver cell fate. Cell. 2014;157(6):1324-1338.

10. Yu FX, Zhao B, Guan KL. Hippo pathway in organ size control, tissue homeostasis, and cancer. Cell. 2015;163(4):811-828.
11. Shiojiri N, Takeshita K, Yamasaki H, Iwata T. Suppression of C/EBP alpha expression in biliary cell differentiation from hepatoblasts during mouse liver development. J Hepatol. 2004;41(5):790-798.

12. Bangru S, et al. Alternative splicing rewires Hippo signaling pathway in hepatocytes to promote liver regeneration. Nat Struct Mol Biol. 2018;25(10):928-939.

13. Bhate A, et al. ESRP2 controls an adult splicing programme in hepatocytes to support postnatal liver maturation. Nat Commun. 2015;6:8768.

14. Warzecha CC, Sato TK, Nabet B, Hogenesch JB, Carstens RP. ESRP1 and ESRP2 are epithelial cell-type-specific regulators of FGFR2 splicing. Mol Cell. 2009;33(5):591-601.

15 . Horiguchi K, et al. TGF- $\beta$ drives epithelialmesenchymal transition through $\delta \mathrm{EF} 1-$ mediated downregulation of ESRP. Oncogene. 2012;31(26):3190-3201.

16. Hyun J, Oh SH, Premont RT, Guy CD, Berg CL, Diehl AM. Dysregulated activation of fetal liver programme in acute liver failure. Gut. 2019;68(6):1076-1087.

17. Wands JR, Carter EA, Bucher NL, Isselbacher KJ. Inhibition of hepatic regeneration in rats by acute and chronic ethanol intoxication. Gastroenterology. 1979;77(3):528-531.

18. Duguay L, Coutu D, Hetu C, Joly JG. Inhibition of liver regeneration by chronic alcohol administration. Gut. 1982;23(1):8-13.

19. Yin M, et al. Essential role of tumor necrosis factor alpha in alcohol-induced liver injury in mice. Gastroenterology.1999;117(4):942-952.

20. Karaca G, et al. Role of Fn14 in acute alcoholic steatohepatitis in mice. Am J Physiol Gastrointest Liver Physiol. 2015;308(4):G325-G334.

21. Kawaguchi Y. Sox9 and programming of liver and pancreatic progenitors. J Clin Invest. 2013;123(5):1881-1886.

22. Nakagawa $H$, et al. Loss of liver E-cadherin induces sclerosing cholangitis and promotes carcinogenesis. Proc Natl Acad Sci U S A. 2014;111(3):1090-1095.

23. Zong Y, et al. Notch signaling controls liver development by regulating biliary differentiation. Development. 2009;136(10):1727-1739.

24. Yang N, et al. TAZ induces growth factorindependent proliferation through activation of EGFR ligand amphiregulin. Cell Cycle. 2012;11(15):2922-2930.

25. Zhang H, Pasolli HA, Fuchs E. Yes-associated protein (YAP) transcriptional coactivator functions in balancing growth and differentiation in skin. Proc Natl Acad Sci U S A. 2011;108(6):2270-2275.

26. Zhao B, et al. TEAD mediates YAP-dependent gene induction and growth control. Genes Dev. 2008;22(14):1962-1971.

27. Zhang $\mathrm{H}$, et al. TEAD transcription factors mediate the function of TAZ in cell growth and epithelial-mesenchymal transition. J Biol Chem. 2009;284(20):13355-13362.

28. Li W, et al. YAP transcriptionally regulates COX-2 expression and GCCSysm-4 (G-4), a dual YAP/ COX-2 inhibitor, overcomes drug resistance in colorectal cancer. J Exp Clin Cancer Res. 2017;36(1):144.

29. Warzecha CC, Shen S, Xing Y, Carstens RP. The epithelial splicing factors ESRP1 and ESRP2 positively and negatively regulate diverse types of alternative splicing events. RNA Biol. 2009;6(5):546-562.

30. Tranchevent LC, et al. Identification of protein features encoded by alternative exons using Exon 
Ontology. Genome Res. 2017;27(6):1087-1097.

31. Shapiro IM, et al. An EMT-driven alternative splicing program occurs in human breast cancer and modulates cellular phenotype. PLoS Genet. 2011;7(8):e1002218.

32. Khanova E, et al. Pyroptosis by caspase11/4-gasdermin-D pathway in alcoholic hepatitis in mice and patients. Hepatology. 2018;67(5):1737-1753.

33. Rosenbluh J, et al. $\beta$-Catenin-driven cancers require a YAP1 transcriptional complex for survival and tumorigenesis. Cell. 2012;151(7):1457-1473.

34. Bertola A, Park O, Gao B. Chronic plus binge ethanol feeding synergistically induces neutrophil infiltration and liver injury in mice: a critical role for E-selectin. Hepatology. 2013;58(5):1814-1823.

35. Bertola A, Mathews S, Ki SH, Wang H, Gao B. Mouse model of chronic and binge ethanol feeding (the NIAAA model). Nat Protoc. 2013;8(3):627-637.

36. Kono $\mathrm{H}$, et al. Development of an intragastric enteral model in the mouse: studies of alcohol-induced liver disease using knockout technology. J Hepatobiliary Pancreat Surg. 2000;7(4):395-400.

37. Gao B, Ahmad MF, Nagy LE, Tsukamoto H. Inflammatory pathways in alcoholic steatohepatitis. J Hepatol. 2019;70(2):249-259.

38. Peng WC, et al. Inflammatory cytokine TNF $\alpha$ promotes the long-term expansion of primary hepatocytes in 3D culture. Cell. 2018;175(6):1607-1619.e15.

39. Ravi S, Bade KS, Hasanin M, Singal AK. Ammonia level at admission predicts in-hospital mortality for patients with alcoholic hepatitis. Gastroenterol Rep (Oxf). 2017;5(3):232-236.

40. Aldridge DR, Tranah EJ, Shawcross DL. Pathogenesis of hepatic encephalopathy: role of ammonia and systemic inflammation. J Clin Exp Hepatol. 2015;5(suppl 1):S7-S20.

41. Qvartskhava N, et al. Hyperammonemia in gene-targeted mice lacking functional hepatic glutamine synthetase. Proc Natl Acad Sci U S A. 2015;112(17):5521-5526.

42. Hakvoort TB, et al. Pivotal role of glutamine synthetase in ammonia detoxification. Hepatology. 2017;65(1):281-293.

43. Massafra V, et al. Farnesoid X receptor activation promotes hepatic amino acid catabolism and ammonium clearance in mice. Gastroenterology. 2017;152(6):1462-1476.e10.

44. Northup PG, Caldwell SH. Coagulation in liver disease: a guide for the clinician. Clin Gastroenterol Hepatol. 2013;11(9):1064-1074.

45. Kim W, Kim DJ. Severe alcoholic hepatitis-current concepts, diagnosis and treatment options. World J Hepatol. 2014;6(10):688-695.

46. Du K, et al. Increased glutaminolysis marks active scarring in nonalcoholic steatohepatitis progression [published online December 25,
2019]. Cell Mol Gastroenterol Hepatol. http://doi.org/10.1016/j.jcmgh.2019.12.006.

47. Liu Y, Wen PH, Zhang XX, Dai Y, He Q. Breviscapine ameliorates $\mathrm{CCl} 4$-induced liver injury in mice through inhibiting inflammatory apoptotic response and ROS generation. Int J Mol Med. 2018;42(2):755-768.

48. Murugan R, et al. Acute kidney injury in nonsevere pneumonia is associated with an increased immune response and lower survival. Kidney Int. 2010;77(6):527-535.

49. Cunningham PN, Dyanov HM, Park P, Wang J, Newell KA, Quigg RJ. Acute renal failure in endotoxemia is caused by TNF acting directly on TNF receptor-1 in kidney. J Immunol. 2002;168(11):5817-5823.

50. Arora R, Kathuria S, Jalandhara N. Acute renal dysfunction in patients with alcoholic hepatitis. World J Hepatol. 2011;3(5):121-124.

51. Zhang W, Sun Q, Zhong W, Sun X, Zhou Z. Hepatic peroxisome proliferator-activated receptor gamma signaling contributes to alcohol-induced hepatic steatosis and inflammation in mice. Alcohol Clin Exp Res. 2016;40(5):988-999.

52. Sato K, Marzioni M, Meng F, Francis H, Glaser S, Alpini G. Ductular reaction in liver diseases: pathological mechanisms and translational significances. Hepatology. 2019;69(1):420-430.

53. Hughes E, Hopkins LJ, Parker R. Survival from alcoholic hepatitis has not improved over time. PLoS ONE. 2018;13(2):e0192393.

54. Nguyen TA, DeShazo JP, Thacker LR, Puri P, Sanyal AJ. The worsening profile of alcoholic hepatitis in the United States. Alcohol Clin Exp Res. 2016;40(6):1295-1303.

55. Mathurin P, et al. Early liver transplantation for severe alcoholic hepatitis. $N$ Engl J Med. 2011;365(19):1790-1800.

56. Liangpunsakul S. Clinical characteristics and mortality of hospitalized alcoholic hepatitis patients in the United States. J Clin Gastroenterol. 2011;45(8):714-719.

57. Hoang SA, et al. Gene expression predicts histological severity and reveals distinct molecular profiles of nonalcoholic fatty liver disease. $S c i$ Rep. 2019;9(1):12541.

58. Mizutani A, Koinuma D, Seimiya H, Miyazono K. The Arkadia-ESRP2 axis suppresses tumor progression: analyses in clear-cell renal cell carcinoma. Oncogene. 2016;35(27):3514-3523.

59. Bebee TW, et al. The splicing regulators Esrp1 and Esrp2 direct an epithelial splicing program essential for mammalian development. Elife. 2015;4:e08954.

60. Neuman MG, et al. Alcoholic liver disease: role of cytokines. Biomolecules. 2015;5(3):2023-2034.

61. Bishehsari F, et al. Alcohol and gut-derived inflammation. Alcohol Res. 2017;38(2):163-171.

62. Fry DE. Sepsis, systemic inflammatory response, and multiple organ dysfunction: the mystery con- tinues. Am Surg. 2012;78(1):1-8.

63. O’Beirne J, Patch D, Holt S, Hamilton M, Burroughs AK. Alcoholic hepatitis-the case for intensive management. Postgrad Med J. 2000;76(898):504-507.

64. Ginès $\mathrm{A}$, et al. Incidence, predictive factors, and prognosis of the hepatorenal syndrome in cirrhosis with ascites. Gastroenterology. 1993;105(1):229-236.

65. Dagher L, Moore K. The hepatorenal syndrome. Gut. 2001;49(5):729-737.

66. Sheron N, et al. Circulating and tissue levels of the neutrophil chemotaxin interleukin- 8 are elevated in severe acute alcoholic hepatitis, and tissue levels correlate with neutrophil infiltration. Hepatology. 1993;18(1):41-46.

67. Sheron N, Bird G, Goka J, Alexander G, Williams R. Elevated plasma interleukin- 6 and increased severity and mortality in alcoholic hepatitis. Clin Exp Immunol. 1991;84(3):449-453.

68. De BK, Gangopadhyay S, Dutta D, Baksi SD, Pani A, Ghosh P. Pentoxifylline versus prednisolone for severe alcoholic hepatitis: a randomized controlled trial. World J Gastroenterol. 2009;15(13):1613-1619.

69. Naveau S, et al. A double-blind randomized controlled trial of infliximab associated with prednisolone in acute alcoholic hepatitis. Hepatology. 2004;39(5):1390-1397.

70. Boetticher NC, et al. A randomized, double-blinded, placebo-controlled multicenter tria of etanercept in the treatment of alcoholic hepatitis. Gastroenterology. 2008;135(6):1953-1960.

71. Huang da W, Sherman BT, Lempicki RA. Systematic and integrative analysis of large gene lists using DAVID bioinformatics resources. Nat Protoc. 2009;4(1):44-57.

72. Huang da W, Sherman BT, Lempicki RA. Bioinformatics enrichment tools: paths toward the comprehensive functional analysis of large gene lists. Nucleic Acids Res. 2009;37(1):1-13.

73. Shannon P, et al. Cytoscape: a software environment for integrated models of biomolecular interaction networks. Genome Res. 2003;13(11):2498-2504.

74. Uesugi T, Froh M, Arteel GE, Bradford BU, Thurman RG. Toll-like receptor 4 is involved in the mechanism of early alcohol-induced liver injury in mice. Hepatology. 2001;34(1):101-108.

75. Lazaro R, et al. Osteopontin deficiency does not prevent but promotes alcoholic neutrophilic hepatitis in mice. Hepatology. 2015;61(1):129-140.

76. Rezanejad H, Lock JH, Sullivan BA, Bonner-Weir S. Generation of pancreatic ductal organoids and whole-mount immunostaining of intact organoids. Curr Protoc Cell Biol. 2019;83(1):e82.

77. Butler A, Hoffman P, Smibert P, Papalexi E, Satija R. Integrating single-cell transcriptomic data across different conditions, technologies, and species. Nat Biotechnol. 2018;36(5):411-420. 